\title{
The Socialist People's Libyan Arab Jamahiriya: Statistical Appendix
}

This Statistical Appendix for the The Socialist People's Libyan Arab Jamahiriya was prepared by a staff team of the International Monetary Fund as background documentation for the periodic consultation with the member country. It is based on the information available at the time it was completed on April 5, 2007. The views expressed in this document are those of the staff team and do not necessarily reflect the views of the government of The Socialist People's Libyan Arab Jamahiriya or the Executive Board of the IMF.

The policy of publication of staff reports and other documents by the IMF allows for the deletion of market-sensitive information.

To assist the IMF in evaluating the publication policy, reader comments are invited and may be sent by e-mail to publicationpolicy@imf.org.

Copies of this report are available to the public from

International Monetary Fund $\bullet$ Publication Services

700 19th Street, N.W. • Washington, D.C. 20431

Telephone: (202) 6237430 • Telefax: (202) 6237201

E-mail: publications@imf.org • Internet: http://www.imf.org

Price: $\$ 18.00$ a copy

\section{International Monetary Fund Washington, D.C.}



INTERNATIONAL MONETARY FUND

\title{
THE SOCIALIST PEOPLE'S LIBYAN ARAB JAMAHIRIYA
}

\author{
Statistical Appendix \\ Prepared by a staff team comprising M. Elhage (head), R. Abdoun, \\ I. Al-Ghelaiqah, and S. Hussein (all MCD) \\ Approved by the Middle East and Central Asia Department
}

April 5, 2007

Contents

Page

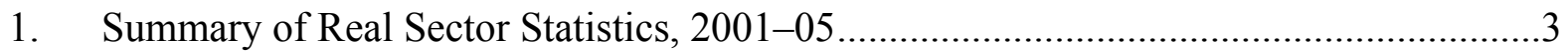

2. Sectoral Distribution of GDP at Current Prices, 2001-05 .............................................

3. Sectoral Distribution of GDP at Constant 1997 Prices, 2001-05 ....................................

4. Gross Fixed Capital Formation by Economic Sector, 2001-05 ......................................6

5. Labor Force and Employment, 2000-04 ………...................................................

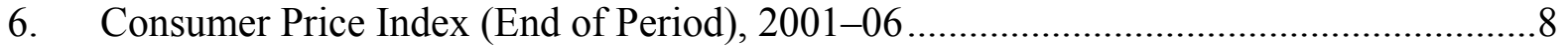

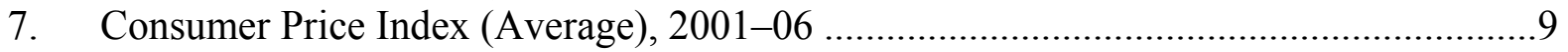

8. Retail Prices of Selected Items in Tripoli, 2001-05 ....................................................

9. Great Man-Made River Financial Operations, 2000-04 ............................................10

10. Domestic Production of Petroleum Products, 2001-06 ..............................................10

11. Domestic Consumption of Petroleum Products, 2001-06 ..............................................11

12. Domestic Retail Prices of Petroleum Products, 2001-06 …...........................................11

13. Production and Exports of Gas and Petrochemicals, 2001-06 .....................................12

14. Production of Crude Oil and Exports of Crude Oil and Refined Products, 2001-06.....12

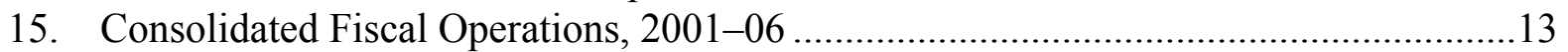

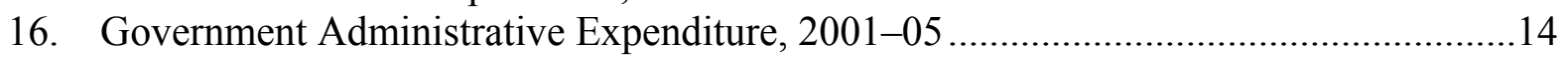

17. Central Government Development Expenditures, 2001-04 ……………….................15

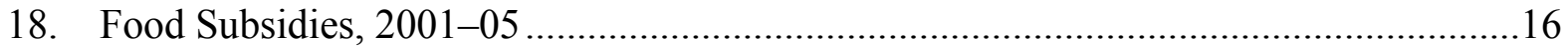

19. Summary Accounts of the Central Bank of Libya, 2001-06 ......................................17

20. Summary Accounts of the Deposit Money Banks and Regional Banks, 2001-06.........18

21. Monetary Survey, 2001-06..................................................................................

22. Foreign Assets and Liabilities of the Central Bank and Commercial Banks, 2001-06..20

23. Summary Accounts of the Libyan Foreign Bank, 2001-06 ……................................21

24. Loans Disbursed by the Agricultural Bank, 2001-06....................................................

25. Loans Disbursed for Housing and Construction Projects by the Savings and Real Estate Investment Bank., 2001-06 ……..............................................................22

26. Loans Disbursed by the Development Bank, 2001-06...............................................22 
27. Distribution of Credit Facilities by Commercial Banks, 2001-06 .............................23

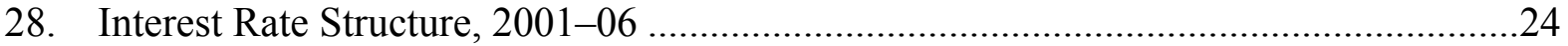

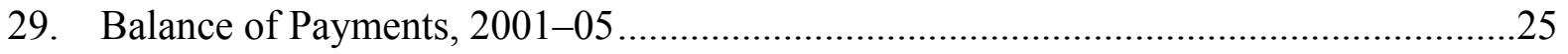

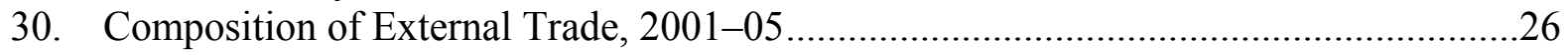

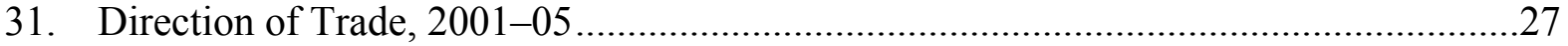

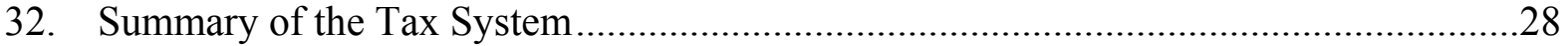


Table 1. Libya: Summary of Real Sector Statistics, 2001-05

\begin{tabular}{|c|c|c|c|c|c|}
\hline & 2001 & 2002 & 2003 & 2004 & 2005 \\
\hline & \multicolumn{5}{|c|}{ (In millions of Libyan dinars) } \\
\hline Nominal GDP at factor costs & 18,745 & 26,837 & 33,077 & 43,067 & 60,040 \\
\hline Nominal hydrocarbon GDP & 7,450 & 14,916 & 20,673 & 29,259 & 44,507 \\
\hline Nominal nonhydrocarbon GDP & 11,295 & 11,920 & 12,403 & 13,808 & 15,533 \\
\hline Nominal GDP at market prices & 19,483 & 25,200 & 30,831 & 39,769 & 54,540 \\
\hline Nominal hydrocarbon GDP & 6,653 & 13,066 & 18,253 & 25,485 & 38,929 \\
\hline Nominal nonhydrocarbon GDP & 12,830 & 12,134 & 12,578 & 14,284 & 15,611 \\
\hline Real GDP at factor cost & 14,036 & 14,235 & 15,080 & 15,831 & 16,828 \\
\hline Real hydrocarbon GDP & 3,701 & 3,409 & 4,013 & 4,310 & 4,669 \\
\hline \multirow[t]{2}{*}{ Real nonhydrocarbon GDP } & 10,335 & 10,825 & 11,066 & 11,521 & 12,159 \\
\hline & \multicolumn{5}{|c|}{ (Annual percentage change) } \\
\hline Nominal GDP at factor costs & 5.7 & 43.2 & 23.3 & 30.2 & 39.4 \\
\hline Nominal hydrocarbon GDP & 5.9 & 100.2 & 38.6 & 41.5 & 52.1 \\
\hline Nominal nonhydrocarbon GDP & 5.6 & 5.5 & 4.1 & 11.3 & 12.5 \\
\hline Nominal GDP at market prices & 5.3 & 29.3 & 22.3 & 29.0 & 37.1 \\
\hline Nominal hydrocarbon GDP & 7.1 & 96.4 & 39.7 & 39.6 & 52.8 \\
\hline Nominal nonhydrocarbon GDP & 4.4 & -5.4 & 3.7 & 13.6 & 9.3 \\
\hline Real GDP at factor costs & 5.9 & 1.4 & 5.9 & 5.0 & 6.3 \\
\hline Real hydrocarbon GDP & 3.3 & -7.9 & 17.7 & 7.4 & 8.3 \\
\hline Real nonhydrocarbon GDP & 6.8 & 4.7 & 2.2 & 4.1 & 5.5 \\
\hline GDP deflator & -0.1 & 41.2 & 16.3 & 24.0 & 31.2 \\
\hline Hydrocarbon deflator & 2.5 & 117.3 & 17.7 & 31.8 & 40.4 \\
\hline Nonhydrocarbon deflator & -1.1 & 0.8 & 1.8 & 6.9 & 6.6 \\
\hline \multicolumn{6}{|l|}{$\mathrm{CPI}$} \\
\hline Average & -8.8 & -9.9 & -2.1 & -2.2 & 2.0 \\
\hline End of period & -10.3 & -7.3 & -1.3 & -3.5 & 3.0 \\
\hline
\end{tabular}

Sources: Libyan authorities; and Fund staff estimates. 
Table 2. Libya: Sectoral Distribution of GDP at Current Prices, 2001-05

\begin{tabular}{|c|c|c|c|c|c|}
\hline & 2001 & 2002 & 2003 & 2004 & 2005 \\
\hline & \multicolumn{5}{|c|}{ (In millions of Libyan dinars) } \\
\hline GDP at factor cost & 18,745 & 26,837 & 33,077 & 43,067 & 60,040 \\
\hline Oil sector & 7,450 & 14,916 & 20,673 & 29,259 & 44,507 \\
\hline Non-oil sector & 11,295 & 11,920 & 12,403 & 13,808 & 15,533 \\
\hline Agriculture, fishing, and forestry & 1,392 & 1,349 & 1,376 & 1,440 & 1,527 \\
\hline Mining & 307 & 387 & 360 & 418 & 459 \\
\hline Manufacturing & 878 & 813 & 765 & 761 & 794 \\
\hline Electricity, gas, and water & 285 & 294 & 303 & 334 & 379 \\
\hline Construction & 1,063 & 1,342 & 1,249 & 1,450 & 1,648 \\
\hline Trade, hotels, and restaurants & 1,882 & 2,090 & 2,205 & 2,418 & 2,859 \\
\hline Transportation, communication, and storage & 1,299 & 1,429 & 1,516 & 1,641 & 1,950 \\
\hline Financing, insurance, and business services & 377 & 415 & 440 & 477 & 564 \\
\hline Housing & 499 & 515 & 534 & 592 & 665 \\
\hline Public services & 2,901 & 2,859 & 3,205 & 3,800 & 4,149 \\
\hline Public services (except education and health) & 1,301 & 1,282 & 1,437 & 1,704 & 1,861 \\
\hline Educational services & 1,035 & 1,020 & 1,143 & 1,355 & 1,480 \\
\hline Health services & 566 & 558 & 625 & 741 & 809 \\
\hline \multirow[t]{2}{*}{ Other services } & 411 & 428 & 451 & 477 & 539 \\
\hline & \multicolumn{5}{|c|}{ (In percent of total) } \\
\hline GDP at factor cost & 100.0 & 100.0 & 100.0 & 100.0 & 100.0 \\
\hline Oil sector & 39.7 & 55.6 & 62.5 & 67.9 & 74.1 \\
\hline Non-oil sector & 60.3 & 44.4 & 37.5 & 32.1 & 25.9 \\
\hline Agriculture, fishing, and forestry & 7.4 & 5.0 & 4.2 & 3.3 & 2.5 \\
\hline Mining & 1.6 & 1.4 & 1.1 & 1.0 & 0.8 \\
\hline Manufacturing & 4.7 & 3.0 & 2.3 & 1.8 & 1.3 \\
\hline Electricity, gas, and water & 1.5 & 1.1 & 0.9 & 0.8 & 0.6 \\
\hline Construction & 5.7 & 5.0 & 3.8 & 3.4 & 2.7 \\
\hline Trade, hotels, and restaurants & 10.0 & 7.8 & 6.7 & 5.6 & 4.8 \\
\hline Transportation, communication, and storage & 6.9 & 5.3 & 4.6 & 3.8 & 3.2 \\
\hline Financing, insurance, and business services & 2.0 & 1.5 & 1.3 & 1.1 & 0.9 \\
\hline Housing & 2.7 & 1.9 & 1.6 & 1.4 & 1.1 \\
\hline Public services & 15.5 & 10.7 & 9.7 & 8.8 & 6.9 \\
\hline \multirow[t]{2}{*}{ Other services } & 2.2 & 1.6 & 1.4 & 1.1 & 0.9 \\
\hline & \multicolumn{5}{|c|}{ (Annual changes in percentage) } \\
\hline GDP at factor cost & 5.7 & 43.2 & 23.3 & 30.2 & 39.4 \\
\hline Oil production & 5.9 & 100.2 & 38.6 & 41.5 & 52.1 \\
\hline Non-oil sector & 5.6 & 5.5 & 4.1 & 11.3 & 12.5 \\
\hline Agriculture, fishing, and forestry & -3.2 & -3.1 & 2.0 & 4.6 & 6.1 \\
\hline Mining & 4.4 & 26.2 & -6.9 & 16.1 & 9.9 \\
\hline Manufacturing & -1.3 & -7.4 & -6.0 & -0.5 & 4.3 \\
\hline Electricity, gas, and water & 5.4 & 3.2 & 3.2 & 10.3 & 13.3 \\
\hline Construction & 4.9 & 26.2 & -6.9 & 16.1 & 13.6 \\
\hline Trade, hotels, and restaurants & 11.7 & 11.0 & 5.5 & 9.7 & 18.3 \\
\hline Transportation, communication, and storage & 7.1 & 10.0 & 6.1 & 8.3 & 18.8 \\
\hline Financing, insurance, and business services & 5.6 & 10.0 & 6.0 & 8.4 & 18.3 \\
\hline Housing & 4.9 & 3.2 & 3.7 & 10.8 & 12.4 \\
\hline Public services & 8.8 & -1.5 & 12.1 & 18.6 & 9.2 \\
\hline Other services & 5.0 & 4.0 & 5.5 & 5.8 & 12.9 \\
\hline \multicolumn{6}{|l|}{ Memorandum items: } \\
\hline Net indirect taxes and subsidies & 738 & $-1,637$ & $-2,246$ & $-3,298$ & $-5,499$ \\
\hline GDP at market prices & 19,483 & 25,200 & 30,831 & 39,769 & 54,540 \\
\hline
\end{tabular}

Source: Ministry of Planning; and staff estimates. 
Table 3. Libya: Sectoral Distribution of GDP at Constant 1997 Prices, 2001-05

\begin{tabular}{|c|c|c|c|c|c|}
\hline & 2001 & 2002 & 2003 & 2004 & 2005 \\
\hline & \multicolumn{5}{|c|}{ (In millions of Libyan dinars) } \\
\hline GDP at factor cost & 14,036 & 14,235 & 15,080 & 15,831 & 16,828 \\
\hline Oil production & 3,701 & 3,409 & 4,013 & 4,310 & 4,669 \\
\hline Non-oil sector & 10,335 & 10,825 & 11,066 & 11,521 & 12,159 \\
\hline Agriculture, fishing, and forestry & 1,322 & 1,357 & 1,384 & 1,411 & 1,447 \\
\hline Mining & 263 & 316 & 294 & 309 & 331 \\
\hline Manufacturing & 746 & 727 & 691 & 680 & 692 \\
\hline Electricity, gas, and water & 316 & 326 & 336 & 353 & 374 \\
\hline Construction & 940 & 1,130 & 1,051 & 1,104 & 1,183 \\
\hline Trade, hotels, and restaurants & 1,698 & 1,774 & 1,863 & 1,975 & 2,123 \\
\hline Transportation, communication, and storage & 1,290 & 1,419 & 1,490 & 1,565 & 1,690 \\
\hline Financing, insurance, and business services & 322 & 354 & 374 & 394 & 424 \\
\hline Housing & 493 & 508 & 525 & 545 & 583 \\
\hline Public services & 2,579 & 2,542 & 2,657 & 2,763 & 2,860 \\
\hline \multirow[t]{2}{*}{ Other services } & 368 & 372 & 401 & 421 & 451 \\
\hline & \multicolumn{5}{|c|}{ (In percent of total) } \\
\hline GDP at factor cost & 100.0 & 100.0 & 100.0 & 100.0 & 100.0 \\
\hline Oil production & 26.4 & 23.9 & 26.6 & 27.2 & 27.7 \\
\hline Non-oil sector & 73.6 & 76.1 & 73.4 & 72.8 & 72.3 \\
\hline Agriculture, fishing, and forestry & 9.4 & 9.5 & 9.2 & 8.9 & 8.6 \\
\hline Mining & 1.9 & 2.2 & 2.0 & 2.0 & 2.0 \\
\hline Manufacturing & 5.3 & 5.1 & 4.6 & 4.3 & 4.1 \\
\hline Electricity, gas, and water & 2.3 & 2.3 & 2.2 & 2.2 & 2.2 \\
\hline Construction & 6.7 & 7.9 & 7.0 & 7.0 & 7.0 \\
\hline Trade, hotels, and restaurants & 12.1 & 12.5 & 12.4 & 12.5 & 12.6 \\
\hline Transportation, communication, and storage & 9.2 & 10.0 & 9.9 & 9.9 & 10.0 \\
\hline Financing, insurance, and business services & 2.3 & 2.5 & 2.5 & 2.5 & 2.5 \\
\hline Housing & 3.5 & 3.6 & 3.5 & 3.4 & 3.5 \\
\hline Public services & 18.4 & 17.9 & 17.6 & 17.5 & 17.0 \\
\hline \multirow[t]{2}{*}{ Other services } & 2.6 & 2.6 & 2.7 & 2.7 & 2.7 \\
\hline & \multicolumn{5}{|c|}{ (Annual changes in percent) } \\
\hline GDP at factor cost & 5.9 & 1.4 & 5.9 & 5.0 & 6.3 \\
\hline Oil production & 3.3 & -7.9 & 17.7 & 7.4 & 8.3 \\
\hline Non-oil sector & 6.8 & 4.7 & 2.2 & 4.1 & 5.5 \\
\hline Agriculture, fishing, and forestry & 3.7 & 2.6 & 2.0 & 2.0 & 2.5 \\
\hline Mining & 4.4 & 20.2 & -7.0 & 5.0 & 7.2 \\
\hline Manufacturing & -4.1 & -2.5 & -5.0 & -1.5 & 1.8 \\
\hline Electricity, gas, and water & 5.4 & 3.2 & 3.2 & 5.0 & 6.0 \\
\hline Construction & 18.1 & 20.2 & -7.0 & 5.0 & 7.2 \\
\hline Trade, hotels, and restaurants & 9.9 & 4.5 & 5.0 & 6.0 & 7.5 \\
\hline Transportation, communication, and storage & 9.5 & 10.0 & 5.0 & 5.0 & 8.0 \\
\hline Financing, insurance, and business services & 41.5 & 10.0 & 5.5 & 5.5 & 7.5 \\
\hline Housing & 4.6 & 3.0 & 3.4 & 3.8 & 7.0 \\
\hline Public services & 2.9 & -1.4 & 4.5 & 4.0 & 3.5 \\
\hline Other services & 4.6 & 1.3 & 7.8 & 5.0 & 7.0 \\
\hline
\end{tabular}

Source: Ministry of Planning; and staff estimates. 
Table 4. Libya: Gross Fixed Capital Formation by Economic Sector, 2001-05

(In millions of Libyan dinars at current prices)

\begin{tabular}{|c|c|c|c|c|c|}
\hline & 2001 & 2002 & 2003 & 2004 & 2005 \\
\hline Agriculture, forestry, and fishing $1 /$ & 479 & 498 & 302 & 252 & 442 \\
\hline Oil and natural gas & 200 & 350 & 385 & 462 & 600 \\
\hline Mining and quarrying & 5 & 10 & 12 & 14 & 15 \\
\hline Manufacturing & 38 & 176 & 147 & 176 & 173 \\
\hline Electricity, gas, and water & 134 & 224 & 600 & 448 & 932 \\
\hline Construction & 9 & 9 & 9 & 11 & 12 \\
\hline Trade, hotels, and restaurants & 11 & 14 & 10 & 21 & 22 \\
\hline Transportation and communication & 242 & 286 & 193 & 432 & 510 \\
\hline Finance, insurance, and real estate & 13 & 9 & 6 & 11 & 15 \\
\hline Ownership of dwellings & 196 & 818 & 818 & 1,274 & 1,231 \\
\hline Public services (including education and housing) & 828 & 1,178 & 840 & 878 & 844 \\
\hline Education & 207 & 318 & $\ldots$ & $\ldots$ & $\ldots$ \\
\hline Health & 120 & 184 & $\ldots$ & $\ldots$ & $\ldots$ \\
\hline Other services & 4 & 7 & 7 & 10 & 11 \\
\hline Total gross fixed capital formation & 2,158 & 3,580 & 3,331 & 3,988 & 4,807 \\
\hline
\end{tabular}

Source: Ministry of Planning.

1/ Includes Great Man-Made River Project. 
Table 5. Libya: Labor Force and Employment, 2000-04 1/

\begin{tabular}{|c|c|c|c|}
\hline Economic Sectors & 2000 & 2001 & 2004 \\
\hline & \multicolumn{3}{|c|}{ (In thousands) } \\
\hline Agriculture, forestry, and fishing & 239.1 & 103.4 & 113.4 \\
\hline Oil and gas extraction & 39.9 & 40.0 & 43.9 \\
\hline Mining and quarrying & 12.5 & 28.9 & 31.7 \\
\hline Manufacturing & 169.6 & 172.1 & 188.8 \\
\hline Electricity, gas, and water & 41.0 & 50.9 & 55.8 \\
\hline Construction & 222.0 & 45.2 & 49.6 \\
\hline Trade, hotels, and restaurants & 69.5 & 161.2 & 176.8 \\
\hline Transportation and communication & 143.4 & 55.5 & 60.9 \\
\hline Finance, insurance, and real estate & 33.0 & 38.1 & 41.8 \\
\hline Public administrations & 118.9 & 219.2 & 240.5 \\
\hline Education services & 198.2 & 365.5 & 401.0 \\
\hline Health services & 86.1 & 158.3 & 173.7 \\
\hline Other services & 71.8 & 20.1 & 22.1 \\
\hline Total employment & $1,445.0$ & $1,458.4$ & $1,600.0$ \\
\hline \multicolumn{4}{|l|}{ Of which: } \\
\hline Libyans & $1,257.1$ & $1,335.4$ & $1,543.1$ \\
\hline \multirow[t]{2}{*}{ Non-Libyans } & 187.9 & 123.0 & 56.9 \\
\hline & \multicolumn{3}{|c|}{ (Growth rates, in percent 2/) } \\
\hline \multicolumn{4}{|l|}{ Memorandum items: } \\
\hline Total employment & 4.4 & 0.9 & 9.7 \\
\hline \multicolumn{4}{|l|}{ Of which: } \\
\hline Libyans & 4.4 & 6.2 & 15.6 \\
\hline Non-Libyans & 4.4 & -34.5 & -53.7 \\
\hline
\end{tabular}

Source: Libyan authorities.

1/ The GMR is included in agriculture and construction.

2/ For 2004, the growth rate is calculated over 2001. 
Table 6. Libya: Consumer Price Index (End of Period), 2001-06

\begin{tabular}{|c|c|c|c|c|c|c|}
\hline & 2001 & 2002 & 2003 & 2004 & 2005 & 2006 \\
\hline Food, beverages, and tobacco & 82.7 & 77.5 & 78.5 & 73.5 & 76.0 & 85.4 \\
\hline Clothes and shoes & 83.7 & 64.2 & 59.3 & 60.7 & 61.3 & 60.3 \\
\hline Dwellings & 85.0 & 84.8 & 82.3 & 82.7 & 87.5 & 99.1 \\
\hline Furniture (household) & 80.6 & 79.0 & 74.6 & 69.7 & 71.6 & 70.9 \\
\hline Health care & 104.9 & 102.0 & 100.7 & 103.6 & 109.5 & 125.3 \\
\hline Transport & 95.8 & 99.7 & 95.9 & 96.2 & 100.1 & 100.3 \\
\hline Education and entertainment & 96.0 & 83.0 & 85.8 & 82.0 & 81.8 & 79.4 \\
\hline Other miscellaneous services & 88.9 & 79.2 & 80.6 & 78.1 & 77.3 & 78.9 \\
\hline \multirow[t]{2}{*}{ Overall Index } & 85.7 & 79.3 & 78.3 & 75.6 & 77.9 & 83.5 \\
\hline & \multicolumn{6}{|c|}{ (In percentage change) } \\
\hline Food, beverages, and tobacco & -13.8 & -6.3 & 1.3 & -6.4 & 3.4 & 12.4 \\
\hline Clothes and shoes & -11.5 & -23.3 & -7.6 & 2.4 & 1.0 & -1.6 \\
\hline Dwellings & -10.5 & -0.2 & -2.9 & 0.5 & 5.8 & 13.3 \\
\hline Furniture (household) & -7.4 & -2.0 & -5.6 & -6.6 & 2.7 & -1.0 \\
\hline Health care & 0.4 & -2.8 & -1.3 & 2.9 & 5.7 & 14.4 \\
\hline Transport & -1.2 & 4.1 & -3.8 & 0.3 & 4.1 & 0.2 \\
\hline Education and entertainment & -4.5 & -13.5 & 3.4 & -4.4 & -0.2 & -2.9 \\
\hline Other miscellaneous services & -7.2 & -10.9 & 1.8 & -3.1 & -1.0 & 2.1 \\
\hline Overall Index & -10.2 & -7.5 & -1.3 & -3.4 & 3.0 & 7.2 \\
\hline
\end{tabular}

Source: Census and Statistics Department. 
Table 7. Libya: Consumer Price Index (Average), 2001-06

\begin{tabular}{|c|c|c|c|c|c|c|}
\hline \multicolumn{7}{|c|}{$(1999=100)$} \\
\hline & 2001 & 2002 & 2003 & 2004 & 2005 & 2006 \\
\hline Food, beverages, and tobacco & 87.8 & 77.1 & 76.8 & 74.8 & 76.6 & 78.8 \\
\hline Clothes and shoes & 83.0 & 65.6 & 60.9 & 60.8 & 61.3 & 61.6 \\
\hline Dwellings & 86.8 & 87.1 & 83.3 & 83.2 & 86.9 & 99.1 \\
\hline Furniture (household) & 82.1 & 79.2 & 76.9 & 70.9 & 71.2 & 71.1 \\
\hline Health care & 106.6 & 101.4 & 97.0 & 103.5 & 107.6 & 115.7 \\
\hline Transport & 96.6 & 99.3 & 99.0 & 95.8 & 98.3 & 100.1 \\
\hline Education and entertainment & 97.0 & 85.1 & 83.5 & 82.2 & 82.4 & 80.8 \\
\hline Other miscellaneous services & 92.2 & 81.7 & 80.3 & 78.7 & 77.5 & 80.3 \\
\hline \multirow[t]{2}{*}{ Overall Index } & 88.5 & 79.8 & 78.1 & 76.4 & 77.9 & 80.6 \\
\hline & \multicolumn{4}{|c|}{ (In percentage change) } & & \\
\hline Food, beverages, and tobacco & -10.0 & -12.2 & -0.4 & -2.5 & 2.3 & 2.9 \\
\hline Clothes and shoes & -11.4 & -21.0 & -7.2 & -0.1 & 0.9 & 0.4 \\
\hline Dwellings & -11.3 & 0.4 & -4.4 & -0.1 & 4.4 & 14.1 \\
\hline Furniture (household) & -13.8 & -3.6 & -2.9 & -7.7 & 0.4 & -0.1 \\
\hline Health care & 5.6 & -4.9 & -4.3 & 6.7 & 3.9 & 7.6 \\
\hline Transport & -1.6 & 2.8 & -0.2 & -3.3 & 2.6 & 1.9 \\
\hline Education and entertainment & -2.5 & -12.3 & -1.9 & -1.5 & 0.2 & -1.9 \\
\hline Other miscellaneous services & -6.0 & -11.4 & -1.8 & -1.9 & -1.6 & 3.6 \\
\hline Overall Index & -8.9 & -9.8 & -2.1 & -2.2 & 2.0 & 3.4 \\
\hline
\end{tabular}

Source: Census and Statistics Department.

1/ Based on a 1992 household expenditure survey.

Table 8. Libya: Retail Prices of Selected Items in Tripoli, 2001-05

(In Libyan dinars)

\begin{tabular}{|c|c|c|c|c|c|c|}
\hline & Unit & 2001 & 2002 & 2003 & 2004 & $\frac{\text { Sept. }}{2005}$ \\
\hline Poultry & Kilogram & 3.147 & 2.812 & 2.634 & 2.636 & 2.308 \\
\hline Eggs & 30 eggs & 3.170 & 2.654 & 2.897 & 2.202 & 3.354 \\
\hline Potatoes & Kilogram & 0.625 & 0.815 & 0.647 & 0.742 & 1.313 \\
\hline Sugar & Kilogram & 0.120 & 0.140 & 0.133 & 0.140 & 0.140 \\
\hline Tea (red) & Kilogram & 1.000 & 1.250 & 1.250 & 1.250 & 1.500 \\
\hline Coffee & Kilogram & 4.000 & 4.000 & 4.000 & 4.000 & 6.000 \\
\hline Olive oil & Liter & 2.009 & 2.281 & 2.396 & 2.500 & 2.333 \\
\hline Sunflower oil & Liter & 0.450 & 0.500 & 0.538 & 0.584 & 0.625 \\
\hline Tobacco & 20 cigarettes & 0.900 & 0.713 & 0.650 & 0.650 & 1.000 \\
\hline Gasoline & Liter & 0.140 & 0.150 & 0.150 & 0.120 & 0.140 \\
\hline Kerosene & Liter & 0.060 & 0.070 & 0.060 & 0.060 & 0.080 \\
\hline Gas in cylinder & 15 Kilograms & 1.250 & 1.250 & 0.125 & 1.250 & 1.500 \\
\hline Electricity & Kilowatts & 0.020 & 0.020 & 0.020 & 0.020 & 0.020 \\
\hline
\end{tabular}

Source: Census and Statistics Department. 
Table 9. Libya: Great Man-Made River Financial Operations, 2000-04

(In millions of Libyan dinars)

\begin{tabular}{lrrrr}
\hline Year & Revenues & Expenditures & $\begin{array}{r}\text { Deficit (-) } \\
\text { Surplus (+) }\end{array}$ & $\begin{array}{r}\text { Accumulated } \\
\text { Deficit/Surplus }\end{array}$ \\
\hline 2000 & 375.5 & 365.9 & 9.6 & -894.9 \\
2001 & 447.7 & 350.5 & 97.2 & -797.7 \\
2002 & 883.9 & 853.8 & 30.1 & -767.6 \\
2003 & 699.3 & 627.7 & 71.6 & -696.0 \\
2004 & 637.0 & 708.0 & -71.0 & -767.0 \\
\hline
\end{tabular}

Source: Great Man-Made River Authority.

Table 10. Libya: Domestic Production of Petroleum Products, 2001-06

(In thousands of metric tons)

\begin{tabular}{lrrrrrr}
\hline & & & & & & Jan.-June \\
\cline { 5 - 7 } & 2001 & 2002 & 2003 & 2004 & 2005 & 2006 \\
\hline Natural gas (bottled) & 163 & 119 & 195 & 191 & 166.7 & 95.8 \\
Gasoline & 757 & 716 & 858 & 654 & 800 & 379.5 \\
Jet fuel & 1,519 & 1,487 & 1,562 & 1,406 & 1495.2 & 712.5 \\
Naphtha (raw) & 2,419 & 2,348 & 2,585 & 2,474 & 2769.8 & 1380.1 \\
Gas oil & 3,743 & 3,352 & 3,771 & 3,842 & 4103.6 & 2059.7 \\
Fuel oil & 6,045 & 5,887 & 6,244 & 6,431 & 7083.8 & 3492.5 \\
$\quad$ Total & 14,646 & 13,909 & 15,216 & 14,999 & 16419.1 & 8120.1 \\
\hline
\end{tabular}

Source: National Oil Corporation. 
Table 11. Libya: Domestic Consumption of Petroleum Products, 2001-06

(In thousands of metric tons)

\begin{tabular}{lrrrrrr}
\hline & 2001 & 2002 & 2003 & 2004 & 2005 & 2006 \\
\hline Gasoline & 1,884 & 2,017 & 2,146 & 2,257 & 2,331 & 1,192 \\
Kerosene and jet fuel & 326 & 325 & 392 & 533 & 632.5 & 270.6 \\
$\quad$ Kerosene & 94 & $\ldots$ & $\ldots$ & $\ldots$ & $\ldots$ & $\ldots$ \\
Jet fuel & 232 & $\ldots$ & $\ldots$ & $\ldots$ & $\ldots$ & $\ldots$ \\
Fuel oil & 1,896 & 2,373 & 2,549 & 2,473 & 2460.2 & 1266.3 \\
Gas oil & 2,660 & 3,073 & 3,429 & 3,783 & 3574.9 & 1816.2 \\
LPG & 256 & 277 & 272 & 281 & 286.7 & 144.5 \\
Asphalt & 55 & 77 & 0 & $\ldots$ & $\ldots$ & $\ldots$ \\
Total & 7,077 & 8,142 & 8,787 & 9,327 & 9285.3 & 4689.2 \\
\hline
\end{tabular}

Source: National Oil Corporation.

Table 12. Libya: Domestic Retail Prices of Petroleum Products, 2001-06 (In dirhams per liter, unless otherwise specified)

\begin{tabular}{lrrrrrr}
\hline & 2001 & 2002 & 2003 & 2004 & 2005 & $\begin{array}{r}\text { Jan.-June } \\
\text { Premium gasoline 1/ } \\
\quad(98 \text { octane premium) }\end{array}$ \\
$\begin{array}{lrrrr}\text { Premium gasoline } \\
\quad(94 \text { octane premium) }\end{array}$ & 140 & 150 & 150 & 150 & $\ldots$ & $\ldots$ \\
Kerosene & 105 & 115 & 115 & 115 & 150 & 150 \\
Gas oil & 60 & 70 & 70 & 70 & 80 & 80 \\
Fuel oil (dinars per ton) & 110 & 120 & 120 & 120 & 140 & 140 \\
LPG (per 15-kilo cylinder) & 12 & 27.6 & 27.6 & 27.6 & 27.6 & 27.6 \\
\hline
\end{tabular}

Source: National Oil Corporation.

1/ Since 2006, this quality of gasoline is no longer sold in Libya. 
Table 13. Libya: Production and Exports of Gas and Petrochemicals, 2001-06

\begin{tabular}{|c|c|c|c|c|c|c|}
\hline & & & & & & Jan.-June \\
\hline & 2001 & 2002 & 2003 & 2004 & 2005 & 2006 \\
\hline & \multicolumn{6}{|c|}{ (In billions of cubic feet) } \\
\hline Gas produced & 484 & 464 & 494 & 527 & 765 & 450 \\
\hline Used & 406 & 391 & 365 & 379 & 415 & 201 \\
\hline \multirow[t]{2}{*}{ Flared } & 78 & 73 & 128 & 148 & 350 & 248 \\
\hline & \multicolumn{6}{|c|}{ (In thousands of metric tons) } \\
\hline Petrochemical exports & 1863 & 1819 & 2022 & 1819 & 1860 & 941 \\
\hline Methanol & 592 & 715 & 659 & 600 & 595 & 323 \\
\hline Ammonia & 132 & 152 & 196 & 138 & 129 & 77 \\
\hline Urea & 740 & 718 & 775 & 758 & 702 & 330 \\
\hline
\end{tabular}

Source: National Oil Corporation.

Table 14. Libya: Production of Crude Oil and Exports of Crude Oil and Refined Products, 2001-06

(In millions of barrels)

\begin{tabular}{lrrrrrrr}
\hline & \multicolumn{3}{c}{ Production } & & \multicolumn{3}{c}{ Exports } \\
\cline { 2 - 3 } & $\begin{array}{c}\text { Daily } \\
\text { Average }\end{array}$ & Total & $\begin{array}{c}\text { Change in } \\
\text { Total } \\
\text { (In percent) }\end{array}$ & & $\begin{array}{c}\text { Daily } \\
\text { Average }\end{array}$ & Total & $\begin{array}{c}\text { Change in } \\
\text { Total } \\
\text { (In percent) }\end{array}$ \\
\hline 2001 & 1.416 & 517 & -0.6 & 1.053 & 384 & 1.3 \\
2002 & 1.297 & 474 & -8.4 & & 0.941 & 344 & -10.6 \\
2003 & 1.534 & 560 & 18.3 & 1.184 & 432 & 25.8 \\
2004 & 1.615 & 591 & 5.6 & 1.256 & 460 & 6.4 \\
2005 & 1.693 & 618 & 4.5 & 1.336 & 488 & 6.0 \\
2006 (June) & 1.723 & 314 & $\ldots$ & 1.384 & 253 & $\ldots$ \\
\hline
\end{tabular}

Sources: Secretariat of Oil; National Oil Corporation; and Fund staff estimates. 
Table 15. Libya: Consolidated Fiscal Operations, 2001-06

\begin{tabular}{|c|c|c|c|c|c|c|}
\hline & 2001 & 2002 & 2003 & 2004 & 2005 & 2006 \\
\hline & \multicolumn{6}{|c|}{ (In millions of Libyan dinars) } \\
\hline Total Revenue & 7,891 & 12,850 & 16,614 & 23,272 & 37,413 & 47,088 \\
\hline Hydrocarbon 1/ & 5,296 & 10,150 & 14,506 & 20,141 & 34,764 & 43,566 \\
\hline Nonhydrocarbon 2/ & 2,596 & 2,700 & 2,108 & 3,131 & 2,650 & 3,523 \\
\hline Nonhydrocarbon Tax Revenue & 2,056 & 1,150 & 725 & 1,617 & 1,526 & 1,786 \\
\hline Taxes on income and profits & 381 & 506 & 70 & 309 & 397 & 691 \\
\hline Taxes on international trade & 1,531 & 379 & 385 & 602 & 517 & 527 \\
\hline Other tax revenue & 143 & 266 & 270 & 705 & 611 & 569 \\
\hline Nontax Revenue & 540 & 1,550 & 1,383 & 1,515 & 1,124 & 1,736 \\
\hline Total expenditure and net lending & 8,038 & 10,063 & 13,396 & 17,230 & 19,060 & 21,377 \\
\hline Total Expenditure & 8,038 & 10,063 & 13,396 & 17,129 & 16,640 & 20,797 \\
\hline Current expenditure & 6,226 & 6,724 & 10,564 & 10,195 & 8,245 & 9,693 \\
\hline Administrative budget & 3,537 & 4,183 & 4,228 & 5,611 & 7,166 & 8,219 \\
\hline Expenditure on goods and services & 3,161 & 3,684 & 3,499 & 4,647 & 5,669 & 6,655 \\
\hline Wages and salaries & 2,297 & 2,546 & 2,812 & 3,445 & 4,007 & 4,575 \\
\hline Other purchases of goods and services & 863 & 1,139 & 688 & 1,202 & 1,662 & 2,080 \\
\hline Interest Payments & 75 & 0 & 0 & 0 & 0 & 0 \\
\hline Subsidies and other current transfers & 301 & 499 & 728 & 964 & 1,497 & 1,564 \\
\hline Defense & $\ldots$ & $\ldots$ & $\ldots$ & 894 & 981 & 769 \\
\hline Extrabudgetary current expenditure & 2,689 & 2,541 & 6,336 & 3,690 & 99 & 705 \\
\hline Oil Reserve Fund 3/ & 2,193 & 1,966 & 5,636 & 3,690 & 99 & 705 \\
\hline Defense & 496 & 575 & 700 & $\ldots$ & $\ldots$ & ... \\
\hline Capital expenditure & 1,813 & 3,339 & 2,832 & 6,933 & 8,395 & 11,105 \\
\hline Development budget & 1,539 & 2,936 & 2,204 & 6,135 & 7,570 & 10,079 \\
\hline Extrabudgetary capital expenditure & 274 & 403 & 628 & 798 & 825 & 1,026 \\
\hline Net lending & 0 & 0 & 0 & 102 & 2,420 & 580 \\
\hline Errors and Omissions 4/ & -447 & 1,229 & $-1,333$ & -859 & 1,979 & 213 \\
\hline Overall balance & 300 & 1,559 & 4,552 & 6,901 & 16,375 & 25,498 \\
\hline Nonhydrocarbon balance & $-4,996$ & $-8,592$ & $-9,955$ & $-13,240$ & $-18,389$ & $-18,068$ \\
\hline Domestic Financing & -300 & $-1,559$ & $-4,552$ & $-6,901$ & $-16,375$ & $-25,498$ \\
\hline Banking system & -213 & $-1,134$ & $-4,207$ & $-6,654$ & $-15,877$ & $-19,874$ \\
\hline \multirow[t]{2}{*}{ Nonbank financing 5/ } & -87 & -425 & -344 & -247 & -498 & $-5,624$ \\
\hline & \multicolumn{6}{|c|}{ ( In percent of GDP ) } \\
\hline Total Revenue & 40.5 & 51.0 & 53.9 & 58.5 & 68.6 & 71.2 \\
\hline Hydrocarbon 1/ & 27.2 & 40.3 & 47.1 & 50.6 & 63.7 & 65.9 \\
\hline Nonhydrocarbon 2/ & 13.3 & 10.7 & 6.8 & 7.9 & 4.9 & 5.3 \\
\hline Total Expenditure and net lending & 41.3 & 39.9 & 43.4 & 43.3 & 34.9 & 32.3 \\
\hline Total Expenditure & 41.3 & 39.9 & 43.4 & 43.1 & 30.5 & 31.5 \\
\hline Of which: Current expenditure & 32.0 & 26.7 & 34.3 & 25.6 & 15.1 & 14.7 \\
\hline Wage bill & 11.8 & 10.1 & 9.1 & 8.7 & 7.3 & 6.9 \\
\hline Subsidies & 1.5 & 2.0 & 2.4 & 2.4 & 2.7 & 2.4 \\
\hline Capital expenditure & 9.3 & 13.2 & 9.2 & 17.4 & 15.4 & 16.8 \\
\hline Budgetary expenditure & 26.1 & 28.2 & 20.9 & 31.8 & 28.8 & 28.8 \\
\hline Extra-budgetary expenditure & 15.2 & 11.7 & 22.6 & 11.3 & 1.7 & 2.6 \\
\hline Net lending & 0.0 & 0.0 & 0.0 & 0.3 & 4.4 & 0.9 \\
\hline Errors and omissions & -2.3 & 4.9 & -4.3 & -2.2 & 3.6 & 0.3 \\
\hline \multicolumn{7}{|l|}{ Memorandum item } \\
\hline Nominal GDP (In millions of Libyan Dinars) & 19,483 & 25,200 & 30,831 & 39,769 & 54,540 & 66,112 \\
\hline
\end{tabular}

Sources: Ministry of finance; and staff estimates and projections.

1/ The difference between total oil revenue and the ORF allocations represents the budgetary oil revenues.

2/ Net of income taxes and includes the contributions to the social security fund.

3/ ORF expenditure for 2003 and 2004 includes payments for the Lockerbie Settlement of LD 1,388 million and

LD 1,404 million, respectively.

4/ Corresponds to operations (net) not accounted for.

5/ For 2006, includes exceptional oil receipts (+ LD 1.6 billion in the form of fees paid by some foreign oil companies) and transfers to the Libya Africa Investment Fund ( -LD 6.8 billion). 
Table 16. Libya: Government Administrative Expenditure, 2001-05

(In millions of Libyan dinars)

\begin{tabular}{lrrrrr}
\hline & 2001 & 2002 & 2003 & 2004 & 2005 \\
\hline Expenditure of central secretariats & & & & & \\
General people's Congress & 49 & 43 & 44 & 47 & 48 \\
General people's Committee & 218 & 222 & 204 & 190 & 178 \\
Justice and public security & 202 & 219 & 244 & 284 & 313 \\
Education and scientific research 1/ & 170 & 175 & 196 & 0 & 0 \\
Finance & 133 & 42 & 41 & 47 & 53 \\
Information and culture & 0 & 0 & 0 & 0 & 0 \\
Foreign affairs & 68 & 178 & 175 & 233 & 240 \\
Planning, economy, and trade & 1 & 1 & 1 & 5 & 6 \\
Tourism & 0 & 0 & 0 & 4 & 5 \\
Miscellaneous and Contingencies & 0 & 129 & 119 & 180 & 227 \\
Transfers to public institutions & 85 & 67 & 58 & 72 & 172 \\
Public debt & 255 & 60 & 60 & 60 & 60 \\
Investment expenditure & 100 & 100 & 0 & 0 & 0 \\
Subsidies & 301 & 499 & 480 & 832 & 1,050 \\
Allocations to the regions & 2,198 & 2,042 & 2,140 & 2,687 & 2,665 \\
Others & 0 & 501 & 297 & 336 & 0 \\
Total administrative expenditures & 3,779 & 4,278 & 4,058 & 4,977 & 5,017 \\
Allocations to the regions (in percent of total) & 58 & 48 & 53 & 54 & 53 \\
\hline
\end{tabular}

Source: Secretariat of Finance

1/ Expenditures for 2004 and 2005 are recorded in allocations to the regions . 
Table 17. Libya : Central Government Development Expenditures, 2001-04 ( In millions of Libyan dinars )

\begin{tabular}{|c|c|c|c|c|}
\hline & 2001 & 2002 & 2003 & 2004 \\
\hline Total development budgetary expenditure & 1,539 & 3,702 & 2,050 & 3,581 \\
\hline Goods-producing sectors: & 305 & 553 & 710 & 865 \\
\hline Agriculture, marine, and livestock wealth $1 /$ & 150 & 184 & 124 & 263 \\
\hline Industry and energy & 156 & 369 & 586 & 603 \\
\hline Economic services sectors & 482 & 688 & 293 & 681 \\
\hline Housing and public utilities & 293 & 454 & 195 & 388 \\
\hline Communication and transportation & 190 & 234 & 98 & 293 \\
\hline Social services sectors: & 410 & 1,272 & 679 & 988 \\
\hline Education, establishment, and youth and scientific research & 216 & 739 & 316 & 603 \\
\hline Health and social security & 136 & 406 & 326 & 249 \\
\hline Justice & 43 & 72 & 31 & 91 \\
\hline Information, culture, and tourism & 15 & 55 & 5 & 45 \\
\hline Other sectors & 341 & 1,189 & 368 & 1,046 \\
\hline Economy and trade & 6 & 33 & 3 & 14 \\
\hline Planning and finance & 12 & 106 & 48 & 83 \\
\hline Foreign affaires & 0 & 0 & 10 & 50 \\
\hline Regional development & 104 & 177 & 144 & 183 \\
\hline Great Man-Made River & 0 & 365 & 161 & 154 \\
\hline Human development & 0 & 169 & 0 & 0 \\
\hline Working groups and Excessive Staff Emergency Reserve & 7 & 0 & 0 & 0 \\
\hline Payoff the previous liabilities & 188 & 316 & 0 & 366 \\
\hline Other & 25 & 23 & 3 & 196 \\
\hline
\end{tabular}

Source: High Planning Council.

1/ Including GMR . 
Table 18. Libya: Food Subsidies, 2001-05 1/

(In millions of Libyan dinars)

\begin{tabular}{lrrrrr}
\hline & 2001 & 2002 & 2003 & 2004 & 2005 \\
\hline Wheat & 12 & 99 & -31 & 77 & 0 \\
Flour & 124 & 151 & 338 & 527 & 491 \\
Sugar & 11 & 22 & 39 & 44 & 55 \\
Rice & 8 & 52 & 46 & 104 & 101 \\
Olive and other vegetable oils & -6 & 5 & 99 & 165 & 120 \\
Tea & 18 & 11 & 15 & 31 & 17 \\
Coffee & 0 & 0 & 0 & 0 & 0 \\
Tomato paste & 9 & 9 & 16 & 0 & 0 \\
Dry yeast & 0 & 1 & 4 & 11 & 10 \\
Dry legumes & 0 & 0 & 0 & 0 & 0 \\
Evaporated milk & -7 & 3 & 56 & 147 & 0 \\
Semolina & 0 & 4 & 37 & 48 & 37 \\
Miscellaneous & 4 & 2 & 6 & 6 & 7 \\
Pasta & 0 & 0 & 0 & 42 & 0 \\
Total & 172 & 357 & 625 & 1,202 & 839 \\
\hline
\end{tabular}

Source : National Supply Corporation (NASCO).

1/ A minus sign indicates an operating surplus. 
Table 19. Libya: Summary Accounts of the Central Bank of Libya, 2001-06

(In millions of Libyan dinars)

\begin{tabular}{|c|c|c|c|c|c|c|}
\hline & 2001 & 2002 & 2003 & 2004 & 2005 & 2006 \\
\hline Foreign assets & 9,414 & 18,444 & 26,578 & 33,073 & 54,460 & 77,253 \\
\hline Gold & 58 & 58 & 58 & 58 & 58 & 58 \\
\hline Foreign exchange & 8,675 & 16,997 & 24,866 & 31,332 & 52,681 & 75,420 \\
\hline Reserve Position with the Fund & 324 & 651 & 764 & 764 & 764 & 764 \\
\hline SDR holdings & 358 & 738 & 890 & 919 & 956 & 1,011 \\
\hline Claims on government & 7,151 & 7,010 & 7,012 & 828 & 828 & 828 \\
\hline Claims on treasury $1 /$ & 6,789 & 6,790 & 6,792 & 608 & 608 & 608 \\
\hline Great Man-Made River (GMR) & 362 & 220 & 220 & 220 & 220 & 220 \\
\hline Claims on other sectors $2 /$ & 339 & 884 & 1,300 & 1,499 & 2,522 & 2,837 \\
\hline Claims on nonfinancial public enterprises & 330 & 873 & 1,287 & 1,485 & 2,505 & 2,819 \\
\hline Claims on private sector & 10 & 11 & 13 & 15 & 16 & 18 \\
\hline Claims on other banking institutions & 0 & 0 & 0 & 0 & 0 & 0 \\
\hline Claims on nonbank financial institutions & 0 & 0 & 0 & 0 & 0 & 0 \\
\hline Claims on deposit money banks & 87 & 58 & 29 & 1 & 2 & 62 \\
\hline Unclassified assets & 648 & 526 & 1,109 & 1,598 & 1,390 & 3,488 \\
\hline Total Assets & 17,639 & 26,921 & 36,028 & 36,999 & 59,201 & 84,468 \\
\hline Total Liabilities & 17,639 & 26,921 & 36,028 & 36,999 & 59,201 & 84,468 \\
\hline Reserve money & 6,141 & 6,240 & 6,891 & 9,966 & 14,423 & 16,657 \\
\hline Currency outside CBL & 2,690 & 2,752 & 2,883 & 2,794 & 3,482 & 4,134 \\
\hline Currency with DMBs & 131 & 138 & 120 & 182 & 173 & 214 \\
\hline Currency outside DMBs & 2,560 & 2,614 & 2,764 & 2,613 & 3,309 & 3,920 \\
\hline Commercial Banks'reserves with CBL & 1,183 & 906 & 914 & 1,510 & 2,748 & 3,487 \\
\hline Demand deposits $3 /$ & 454 & 460 & 351 & 740 & 1,407 & 1,016 \\
\hline Time and savings deposits & 1,813 & 2,123 & 2,743 & 4,921 & 6,786 & 8,020 \\
\hline Of which: Commercial Banks & 1,614 & 1,984 & 2,634 & 4,876 & 6,752 & 2,040 \\
\hline Restricted deposits & 738 & 1,468 & 1,702 & 1,352 & 1,549 & 2,608 \\
\hline Government deposits & 7,765 & 8,587 & 12,964 & 19,519 & 32,563 & 53,721 \\
\hline Treasury deposits & 7,384 & 8,217 & 12,551 & 19,105 & 31,890 & 52,588 \\
\hline Social Security Fund deposits & 381 & 370 & 413 & 414 & 673 & 1,133 \\
\hline Foreign liabilities & 4 & 4 & 5 & 7 & 12 & 13 \\
\hline Capital accounts & 2,639 & 9,919 & 13,991 & 4,315 & 5,761 & 5,002 \\
\hline Unclassified liabilities & 352 & 702 & 476 & 1,841 & 4,894 & 6,469 \\
\hline
\end{tabular}

Source: Central Bank of Libya.

1/ Treasury bonds, bills, advances, and overdrafts.

2/ Largely public sector enterprises.

3/ Almost all belong to public sector enterprises. 
Table 20. Libya: Summary Accounts of the Deposit Money Banks and Regional Banks, 2001-06

(In millions of Libyan dinars)

\begin{tabular}{|c|c|c|c|c|c|c|}
\hline & 2001 & 2002 & 2003 & 2004 & 2005 & 2006 \\
\hline Reserves & 2,447 & 2,557 & 3,258 & 6,342 & 9,204 & 11,506 \\
\hline Currency & 131 & 138 & 120 & 182 & 173 & 214 \\
\hline Deposits with central bank & 2,316 & 2,420 & 3,138 & 6,160 & 9,031 & 11,292 \\
\hline Foreign assets & 623 & 778 & 794 & 1,312 & 2,046 & 2,299 \\
\hline Claims on government & 1,811 & 1,811 & 1,811 & 373 & 373 & 373 \\
\hline Treasury bills & 1,438 & 1,438 & 1,438 & 0 & 0 & 0 \\
\hline Great Man-Made River (GMR) & 373 & 373 & 373 & 373 & 373 & 373 \\
\hline Claims on other sectors & 6,137 & 6,569 & 7,005 & 6,712 & 6,379 & 7,288 \\
\hline Claims on nonfinancial public enterprises & 1,501 & 1,965 & 2,497 & 1,993 & 1,766 & 2,329 \\
\hline Claims on private sector & 4,482 & 4,427 & 4,285 & 4,438 & 4,560 & 4,873 \\
\hline Claims on other banking institutions & 78 & 82 & 77 & 67 & 45 & 75 \\
\hline Claims on nonbank financial institutions & 76 & 96 & 146 & 215 & 7 & 11 \\
\hline Unclassified assets & 1,629 & 1,820 & 1,700 & 1,813 & 1,795 & 2,634 \\
\hline Total Assets & 12,647 & 13,535 & 14,566 & 16,552 & 19,795 & 24,099 \\
\hline Total Liabilities & 12,647 & 13,535 & 14,566 & 16,552 & 19,795 & 24,099 \\
\hline Demand deposits & 4,690 & 5,632 & 5,915 & 7,184 & 9,312 & 11,555 \\
\hline Time, savings, and foreign currency deposits & 2,339 & 2,021 & 2,421 & 2,554 & 3,034 & 3,227 \\
\hline Time and savings deposits & 2,309 & 1,994 & 2,383 & 2,519 & 2,958 & 3,133 \\
\hline Foreign currency deposits & 31 & 28 & 38 & 35 & 76 & 94 \\
\hline Restricted deposits & 741 & 670 & 792 & 857 & 1,176 & 1,415 \\
\hline Foreign liabilities & 57 & 94 & 244 & 141 & 72 & 105 \\
\hline Credit from central bank & 91 & 61 & 32 & 1 & 1 & 58 \\
\hline Government deposits & 582 & 788 & 678 & 1,147 & 1,227 & 1,918 \\
\hline Capital accounts & 992 & 1,306 & 1,470 & 1,566 & 1,844 & 2,006 \\
\hline Unclassified liabilities & 3,155 & 2,962 & 3,016 & 3,102 & 3,130 & 3,816 \\
\hline
\end{tabular}

Source: Central Bank of Libya 
Table 21. Libya: Monetary Survey, 2001-06 1/

\begin{tabular}{|c|c|c|c|c|c|c|}
\hline & 2001 & 2002 & 2003 & 2004 & 2005 & 2006 \\
\hline Net foreign assets & 9,976 & 19,123 & 27,123 & 34,237 & 56,422 & 79,435 \\
\hline Central bank & 9,410 & 18,440 & 26,573 & 33,066 & 54,448 & 77,241 \\
\hline Foreign assets & 9,414 & 18,444 & 26,578 & 33,073 & 54,460 & 77,253 \\
\hline Foreign liabilities & 4 & 4 & 5 & 7 & 12 & 13 \\
\hline Deposit money banks & 566 & 683 & 550 & 1,171 & 1,974 & 2,195 \\
\hline Foreign assets & 623 & 778 & 794 & 1,312 & 2,046 & 2,299 \\
\hline Foreign liabilities & 57 & 94 & 244 & 141 & 72 & 105 \\
\hline Net domestic assets & 1,745 & $-6,119$ & $-13,071$ & $-18,893$ & $-36,600$ & $-55,600$ \\
\hline Domestic credit & 7,092 & 6,899 & 3,486 & $-11,253$ & $-23,688$ & $-44,312$ \\
\hline Net claims on government & 616 & -555 & $-4,820$ & $-19,465$ & $-32,588$ & $-54,438$ \\
\hline Central bank claims & 7,151 & 7,010 & 7,012 & 828 & 828 & 828 \\
\hline Governments' deposits with central bank & 7,765 & 8,587 & 12,964 & 19,519 & 32,563 & 53,721 \\
\hline Commercial banks' claims & 1,811 & 1,811 & 1,811 & 373 & 373 & 373 \\
\hline Governments' deposits with commercial banks & 582 & 788 & 678 & 1,147 & 1,227 & 1,918 \\
\hline Claims on the economy & 6,477 & 7,453 & 8,305 & 8,212 & 8,900 & 10,125 \\
\hline Central bank & 339 & 884 & 1,300 & 1,499 & 2,522 & 2,837 \\
\hline Deposit money banks & 6,137 & 6,569 & 7,005 & 6,712 & 6,379 & 7,288 \\
\hline Claims on nonfinancial public enterprises & 1,831 & 2,838 & 3,784 & 3,477 & 4,271 & 5,148 \\
\hline Claims on private sector & 4,492 & 4,438 & 4,298 & 4,452 & 4,576 & 4,891 \\
\hline Claims on specialized banking institutions & 78 & 82 & 77 & 67 & 45 & 75 \\
\hline Claims on nonbank financial institutions & 76 & 96 & 146 & 215 & 7 & 11 \\
\hline Other items (net) & $-5,347$ & $-13,018$ & $-16,557$ & $-7,640$ & $-12,912$ & $-11,288$ \\
\hline Broad money & 11,721 & 13,004 & 14,052 & 15,344 & 19,822 & 23,835 \\
\hline Money & 7,704 & 8,705 & 9,029 & 10,537 & 14,028 & 16,491 \\
\hline Currency in circulation & 2,560 & 2,614 & 2,764 & 2,613 & 3,309 & 3,920 \\
\hline Demand deposits (other than government) & 5,144 & 6,092 & 6,266 & 7,924 & 10,719 & 12,571 \\
\hline Quasi money & 4,018 & 4,299 & 5,023 & 4,807 & 5,794 & 7,344 \\
\hline Of which: Restricted deposits & 1,479 & 2,138 & 2,494 & 2,208 & 2,725 & 4,023 \\
\hline \multicolumn{7}{|l|}{ Memorandum items: } \\
\hline \multirow[t]{2}{*}{ Net claims on the government excl. SSF 2/ } & 1,057 & -77 & $-4,284$ & $-18,938$ & $-31,815$ & $-53,168$ \\
\hline & \multicolumn{6}{|c|}{ (Annual rate of change in percent) } \\
\hline Broad money & 11.1 & 10.9 & 8.1 & 9.2 & 29.2 & 20.2 \\
\hline Money & 3.6 & 13.0 & 3.7 & 16.7 & 33.1 & 17.6 \\
\hline Quasi money & 28.7 & 7.0 & 16.8 & -4.3 & 20.5 & 26.8 \\
\hline Net claims on Government & -30.6 & -190.1 & 769.0 & 303.9 & 67.4 & 67.0 \\
\hline \multirow[t]{2}{*}{ Claims on nonfinancial public enterprises } & 40.8 & 55.0 & 33.3 & -8.1 & 22.8 & 20.5 \\
\hline & \multicolumn{6}{|c|}{ (Change in percent of beginning of the period money stock) } \\
\hline Net foreign assets & 20.9 & 78.0 & 61.5 & 50.6 & 144.6 & 116.1 \\
\hline Domestic credit & 4.6 & -1.7 & -26.2 & -104.9 & -81.0 & -104.0 \\
\hline Net claims on government & -2.6 & -10.0 & -32.8 & -104.2 & -85.5 & -110.2 \\
\hline Claims on the economy & 7.1 & 8.3 & 6.6 & -0.7 & 4.5 & 6.2 \\
\hline Claims on nonfinancial public enterprises & 5.0 & 8.6 & 7.3 & -2.2 & 5.2 & 4.4 \\
\hline \multirow[t]{2}{*}{ Claims on private sector } & 2.0 & -0.5 & -1.1 & 1.1 & 0.8 & 1.6 \\
\hline & \multicolumn{6}{|c|}{ (As percent of GDP) } \\
\hline Domestic credit & 36.4 & 27.4 & 11.3 & -28.3 & -43.4 & -67.0 \\
\hline Net claims on the government & 3.2 & -2.2 & -15.6 & -48.9 & -59.8 & -82.3 \\
\hline Broad money & 60.2 & 51.6 & 45.6 & 38.6 & 36.3 & 36.1 \\
\hline Nominal GDP (in millions of Libyan dinars) & 19,483 & 25,200 & 30,831 & 39,769 & 54,540 & 66,112 \\
\hline
\end{tabular}

Source: Central Bank of Libya

$1 /$ Data include the regional banks.

2/ The SSF is the Social Security Fund. 
Table 22. Libya: Foreign Assets and Liabilities of the Central Bank and Commercial Banks, 2001-06

\begin{tabular}{|c|c|c|c|c|c|c|}
\hline & 2001 & 2002 & 2003 & 2004 & 2005 & 2006 \\
\hline \multicolumn{7}{|l|}{ Central Bank of Libya } \\
\hline Foreign assets & 9,414 & 18,444 & 26,578 & 33,073 & 54,460 & 77,253 \\
\hline Gold & 58 & 58 & 58 & 58 & 58 & 58 \\
\hline Foreign exchange & 8,675 & 16,997 & 24,866 & 31,332 & 52,681 & 75,420 \\
\hline Reserve position in IMF & 324 & 651 & 764 & 764 & 764 & 764 \\
\hline SDR holdings & 358 & 738 & 890 & 919 & 956 & 1,011 \\
\hline Foreign liabilities & 4 & 4 & 5 & 7 & 12 & 13 \\
\hline Foreign assets (net) & 9,410 & 18,440 & 26,573 & 33,066 & 54,448 & 77,241 \\
\hline \multicolumn{7}{|l|}{ Deposit money banks } \\
\hline Foreign assets & 623 & 778 & 794 & 1,312 & 2,046 & 2,299 \\
\hline Foreign liabilities & 57 & 94 & 244 & 141 & 72 & 105 \\
\hline Foreign assets (net) & 566 & 683 & 550 & 1,171 & 1,974 & 2,195 \\
\hline \multicolumn{7}{|l|}{ Banking system } \\
\hline Foreign assets & 10,037 & 19,221 & 27,371 & 34,385 & 56,505 & 79,552 \\
\hline Foreign liabilities & 61 & 98 & 248 & 148 & 83 & 117 \\
\hline Foreign assets (net) & 9,976 & 19,123 & 27,123 & 34,237 & 56,422 & 79,435 \\
\hline Net foreign assets of central bank & 14,475 & 15,242 & 20,434 & 26,574 & 40,272 & 57,131 \\
\hline Net foreign assets of commercial banks & 870 & 565 & 423 & 941 & 1,460 & 1,623 \\
\hline Net foreign assets of banking system & 15,346 & 15,807 & 20,857 & 27,516 & 41,732 & 58,754 \\
\hline Official exchange rate (LD/US\$, eop) & 0.65 & 1.21 & 1.30 & 1.24 & 1.35 & 1.35 \\
\hline Official exchange rate (LD/US\$, pa) & 0.61 & 1.27 & 1.28 & 1.30 & 1.31 & 1.31 \\
\hline
\end{tabular}

Source: Central Bank of Libya. 
Table 23. Libya: Summary Accounts of the Libyan Foreign Bank, 2001-06

(In millions of Libyan dinars)

\begin{tabular}{|c|c|c|c|c|c|c|}
\hline & 2001 & 2002 & 2003 & 2004 & 2005 & $\frac{\text { Sept. }}{2006}$ \\
\hline Current assets & 3,581 & 6,579 & 7,169 & 9,746 & 10,828 & 16,521 \\
\hline Cash and short-term balances with banks & 259 & 308 & 521 & 505 & 513 & 935 \\
\hline Time deposits with banks & 3,043 & 5,781 & 6,120 & 9,010 & 10,216 & 15,411 \\
\hline Short-term facilities & 279 & 490 & 528 & 231 & 99 & 174 \\
\hline Noncurrent assets & 2,138 & 3,662 & 4,549 & 4,357 & 4,572 & 4,374 \\
\hline Investments, loans, and securities & 1,518 & 2,678 & 3,315 & 3,153 & 3262 & 3708 \\
\hline Participations & 437 & 668 & 790 & 1,072 & 1232 & 617 \\
\hline Other noncurrent assets & 183 & 316 & 445 & 133 & 78 & 48 \\
\hline Unpaid capital & 0 & 0 & 0 & 0 & 0 & 0 \\
\hline Fixed assets & 2 & 3 & 4 & 3 & 2.7 & 2.1 \\
\hline Assets $=$ liabilities & 5,721 & 10,244 & 11,721 & 14,106 & 15,402 & 20,897 \\
\hline Current liabilities & 4,520 & 7,554 & 8,423 & 10,392 & 11,249 & 17,140 \\
\hline Demand deposits & 442 & 1,081 & 1,214 & 1,550 & 1,921 & 1,514 \\
\hline Time deposits & 4,002 & 6,351 & 7,062 & 8,702 & 9,164 & 15,531 \\
\hline Other current liabilities & 76 & 122 & 146 & 141 & 163 & 95 \\
\hline Noncurrent liabilities & 911 & 2,392 & 2,990 & 2,449 & 2,713 & 1,831 \\
\hline Share capital and reserves & 290 & 298 & 309 & 1,265 & 1,441 & 1,926 \\
\hline Shareholders dividend & 0 & 0 & 0 & 0 & 0 & 0 \\
\hline
\end{tabular}

Source: Libyan Foreign Bank.

Table 24. Libya: Loans Disbursed by the Agricultural Bank, 2001-06

(In millions of Libyan dinars)

\begin{tabular}{lrrrrrr}
\hline & & & & & & Sept. \\
\cline { 5 - 6 } & 2001 & 2002 & 2003 & 2004 & 2005 & 2006 \\
\hline Short-term loans & 55.2 & 57.4 & 51.9 & 62.2 & 60.1 & 95 \\
Medium-term loans & 44.5 & 92.4 & 115.6 & 137.1 & 296.5 & 368 \\
Long-term loans & 31.5 & 82.9 & 130.5 & 146.9 & 356.1 & 434 \\
Total new disbursed, year-on-year & 36.5 & 118.5 & 79.9 & 78.6 & $\ldots$ & $\ldots$ \\
Repayment, year-on-year & 18.3 & 17.1 & 14.5 & 6.2 & $\ldots$ & $\ldots$ \\
Loan stock outstanding at end of period & 131.2 & 232.6 & 298.0 & 370.5 & 717 & $\ldots$ \\
\hline
\end{tabular}

Source: Agricultural Bank. 
Table 25. Libya: Loans Disbursed for Housing and Construction Projects by the Savings and Real Estate Investment Bank, 2001-06

(In millions of Libyan dinars)

\begin{tabular}{lrrrrrr}
\hline & & & & & Sept. & \\
\cline { 5 - 6 } & 2001 & $20021 /$ & 2003 & 2004 & 2005 & 2006 \\
\hline Housing loans & 75.5 & 302.9 & 511.6 & 885.5 & $1,855.9$ & $2,711.0$ \\
Construction projects & 18.7 & 12.5 & 198.6 & 83.8 & 95.1 & 203.9 \\
Total new disbursed, year on year & 94.2 & 315.4 & 710.2 & 969.3 & $\ldots$ & $\ldots$ \\
Repayment, year-on-year & 6.0 & 42.2 & 517.1 & $\ldots$ & $\ldots$ & $\ldots$ \\
Loan stock outstanding at end of period & 608.9 & 882.1 & $1,075.2$ & $1,150.4$ & $1,976.5$ & $\ldots$ \\
\hline
\end{tabular}

Source: Savings and Real Estate Investment Bank.

1/ In 2002, includes loans from municipalities (shaabiyat).

Table 26. Libya: Loans Disbursed by the Development Bank, 2001-06

(In millions of Libyan dinars)

\begin{tabular}{lrrrrrr}
\hline & & & & & Sept. \\
\cline { 3 - 6 } & 2001 & 2002 & 2003 & 2004 & 2005 \\
\hline Food industries & 4.8 & 7.4 & 16.7 & 11.2 & 10.8 & 2006 \\
Building materials plastics & 2.1 & 5.1 & 10.9 & 6.5 & 18.6 & 39.4 \\
Chemicals and plastics & 6.7 & 18.8 & 13.7 & 4.1 & 8.6 & 10.6 \\
Metal works & 1.9 & 10.0 & 10.1 & 2.2 & 3.2 & 2.3 \\
Textile industries & 1.3 & 0.2 & 0.8 & 0.2 & 0.2 & 0.0 \\
Furniture industries & 0.7 & 0.3 & 1.1 & 0.3 & 8.4 & 0.2 \\
Industrial services workshops & 5.5 & 17.1 & 16.7 & 7.1 & 196.7 & 115.0 \\
Others & 8.8 & 10.3 & 7.8 & 4.4 & 8.0 & 5.2 \\
Total loans disbursed & 31.7 & 69.2 & 77.8 & 36.0 & 254.4 & 179.0 \\
Loan stock outstanding at end of period & 212.0 & 339.6 & 429.0 & 498.9 & 502.7 & $\ldots$ \\
\hline
\end{tabular}

Source: Development Bank. 
Table 27. Libya: Distribution of Credit Facilities by Commercial Banks, 2001-06 (In millions of Libyan dinars)

\begin{tabular}{|c|c|c|c|c|c|c|}
\hline Sectors & 2001 & 2002 & 2003 & 2004 & 2005 & $\frac{\text { Sept. }}{2006}$ \\
\hline Petroleum & 107 & 169 & 206 & 629 & 501 & 905 \\
\hline Electricity & 347 & 381 & 443 & 101 & 79 & 87 \\
\hline Transportation and communication & 149 & 130 & 95 & 165 & 58 & 109 \\
\hline Planning and economy & 929 & 1,138 & 1,066 & 546 & 460 & 511 \\
\hline Industry & 346 & 318 & 367 & 365 & 218 & 308 \\
\hline Treasury & 7 & 23 & 23 & 12 & 18 & 9 \\
\hline Health & 133 & 85 & 203 & 162 & 42 & 39 \\
\hline Agriculture & 172 & 191 & 232 & 229 & 106 & 102 \\
\hline Housing and public utilities & 472 & 439 & 411 & 451 & 261 & 213 \\
\hline Education & 13 & 39 & 44 & 40 & 46 & 52 \\
\hline Information and culture & 8 & 12 & 34 & 35 & 14 & 15 \\
\hline Social security & 4 & 1 & 4 & 2 & 0 & 1 \\
\hline Tourism & 49 & 57 & 61 & 50 & 38 & 47 \\
\hline Marine wealth & 39 & 44 & 39 & 31 & 29 & 6 \\
\hline Justice and general security & 15 & 16 & 22 & 54 & 17 & 24 \\
\hline Great Man-Made River & 394 & 421 & 420 & 414 & 373 & 373 \\
\hline National Investment Council & 89 & 0 & 0 & 0 & 0 & 0 \\
\hline National Banking corporation & 31 & 31 & 29 & 19 & 19 & 18 \\
\hline Specialized banks & 38 & 25 & 44 & 44 & 6 & 5 \\
\hline Craftsmen and partnerships & 60 & 0 & 0 & 0 & 0 & 0 \\
\hline Other private sector & 359 & 0 & 0 & 0 & 0 & 0 \\
\hline Social loans & 1,083 & 1,699 & 1,959 & 1,487 & 1,426 & 1,402 \\
\hline Housing loans & 1,469 & 1,103 & 1,165 & 1,456 & 591 & 517 \\
\hline Other domestic & 582 & 945 & 544 & 920 & $\cdots$ & $\cdots$ \\
\hline Total domestic & 6,896 & 7,266 & 7,411 & 7,212 & 5,997 & 6,507 \\
\hline Foreign companies & 151 & 195 & 269 & 198 & 83 & 105 \\
\hline Foreign letters of guarantees & 0 & 0 & 0 & 0 & 0 & 0 \\
\hline Total domestic and foreign & 7,047 & 7,461 & 7,680 & 7,410 & 6,167 & 6,723 \\
\hline
\end{tabular}

Source: Central Bank of Libya. 
Table 28. Libya: Interest Rate Structure, 2000-06

( In percent )

\begin{tabular}{|c|c|c|c|c|c|}
\hline & 2000-02 & 2003 & 2004 & 2005 & 2006 \\
\hline \multicolumn{6}{|l|}{ Central bank and treasury } \\
\hline Rediscount rate & 5.00 & 5.00 & 4.00 & 4.00 & 4.00 \\
\hline Treasury bills rate $1 /$ & 5.00 & 5.50 & 5.50 & $\ldots$ & $\ldots$ \\
\hline Treasury bonds rate $1 /$ & 5.00 & 6.00 & 6.00 & $\ldots$ & $\ldots$ \\
\hline \multicolumn{6}{|l|}{ Commercial banks : } \\
\hline \multicolumn{6}{|l|}{ Deposit rates (actuals) } \\
\hline \multicolumn{6}{|l|}{ Fixed term deposits rates } \\
\hline 10 days or less & 2.50 & 2.50 & 1.50 & $\ldots$ & $\ldots$ \\
\hline $11-30$ days & 3.00 & 3.00 & 2.00 & $\ldots$ & $\ldots$ \\
\hline $31-89$ days & 3.50 & 3.50 & 2.50 & $\ldots$ & $\ldots$ \\
\hline $90-180$ days & 4.00 & 4.00 & 3.00 & $\ldots$ & $\ldots$ \\
\hline $181-360$ days & 5.00 & 5.00 & 4.00 & $\ldots$ & $\ldots$ \\
\hline One year & 5.50 & 5.50 & 4.50 & $\ldots$ & $\ldots$ \\
\hline Two years & 5.50 & 5.50 & 4.50 & $\ldots$ & $\ldots$ \\
\hline Three years & 5.50 & 5.50 & 4.50 & $\ldots$ & $\ldots$ \\
\hline Four years and over & 5.50 & 5.50 & 4.50 & $\cdots$ & $\cdots$ \\
\hline \multicolumn{6}{|l|}{ Savings deposits rates } \\
\hline LD 0-20,000 & 6.00 & 6.00 & 5.00 & $\ldots$ & $\ldots$ \\
\hline LD 20,000-100,000 & 5.00 & 5.00 & 4.00 & $\ldots$ & $\ldots$ \\
\hline LD 100,000 and more & 0.00 & 0.00 & 0.00 & $\ldots$ & ... \\
\hline \multicolumn{6}{|l|}{$\begin{array}{l}\text { Lending rates (maximum) 2/ } \\
\text { Loans and advances }\end{array}$} \\
\hline Secured & 7.00 & 7.00 & 6.00 & 6.50 & 6.50 \\
\hline Unsecured & 7.50 & 7.50 & 6.50 & 6.50 & 6.50 \\
\hline Agricultural, industrial & 6.00 & 6.00 & 3.00 & 6.50 & 6.50 \\
\hline Real estate loans & 2.00 & 3.00 & 3.00 & 6.50 & 6.50 \\
\hline Civil servants to production & 5.00 & 3.00 & 3.00 & 6.50 & 6.50 \\
\hline Interbank lending rates & 4.00 & 4.00 & 4.00 & 4.00 & 4.00 \\
\hline
\end{tabular}

Source : Central Bank of Libya

$1 /$ In 2004, the government bought back its entire debt from the banking system.

2/ As of August 2005, banks were granted autonomy to freely determine interest rates on deposits and to set lending rates within a band of 250 basis points above the discount rate. 
Table 29. Libya: Balance of Payments, 2001-05

(In millions of US dollars)

\begin{tabular}{|c|c|c|c|c|c|}
\hline & 2001 & 2002 & 2003 & 2004 & $\begin{array}{c}\text { Prel. } \\
2005\end{array}$ \\
\hline Current Account & 4,267 & 652 & 5,158 & 7,410 & 17,325 \\
\hline Goods and Services & 5,338 & 1,259 & 6,292 & 10,165 & 18,240 \\
\hline Goods & 6,188 & 2,395 & 7,447 & 11,642 & 20,072 \\
\hline Exports (fob) & 11,014 & 9,803 & 14,647 & 20,410 & 30,948 \\
\hline Hydrocarbon sector 1/ & 10,594 & 9,620 & 14,159 & 19,533 & 30,448 \\
\hline Other exports & 420 & 184 & 489 & 877 & 500 \\
\hline Imports (fob) & $-4,825$ & $-7,408$ & $-7,200$ & $-8,768$ & $-10,875$ \\
\hline Of which: oil sector & -752 & -626 & -950 & $-1,271$ & $-1,378$ \\
\hline Services (net) & -850 & $-1,137$ & $-1,155$ & $-1,477$ & $-1,832$ \\
\hline Debit & 1,033 & 1,539 & 1,597 & 1,914 & 2,320 \\
\hline Credit & 183 & 402 & 442 & 437 & 488 \\
\hline Freight and insurance for imports & -263 & -343 & -540 & -658 & $\ldots$ \\
\hline Travel & -494 & -628 & -352 & -368 & $\ldots$ \\
\hline Transportation & -169 & -83 & -104 & -85 & $\ldots$ \\
\hline Government services (other) & -209 & -190 & 43 & 45 & $\ldots$ \\
\hline Private services (other) & -79 & -100 & -202 & -134 & $\ldots$ \\
\hline Of which: Hydrocarbon sector & -49 & -23 & -23 & -33 & $\ldots$ \\
\hline Income & -240 & 265 & 540 & -246 & -281 \\
\hline Direct investment income 2/ & -747 & -585 & -845 & $-1,337$ & $-1,834$ \\
\hline Other investment income & 507 & 850 & 1,385 & 1,091 & 1,553 \\
\hline Government sector & 414 & 740 & 1,263 & 966 & 1,403 \\
\hline Private sector & 93 & 110 & 122 & 125 & 150 \\
\hline Current transfers & -832 & -872 & $-1,673$ & $-2,509$ & -634 \\
\hline General government 3/ & 0 & 0 & $-1,174$ & $-1,741$ & -112 \\
\hline Private sector & -832 & -872 & -499 & -768 & -522 \\
\hline Oil sector & -127 & -105 & -156 & -210 & -259 \\
\hline Other sectors (workers transfers abroad) & -705 & -767 & -343 & -558 & -588 \\
\hline Capital and Financial Account & $-2,073$ & 78 & -316 & $-1,830$ & 72 \\
\hline Direct investment & -308 & 281 & 79 & -643 & 1,499 \\
\hline Abroad & -175 & 136 & -63 & $-1,000$ & -128 \\
\hline In Libya & -133 & 145 & 142 & 357 & 1,627 \\
\hline Portfolio investment & -393 & -393 & -393 & -393 & -393 \\
\hline Other investment & -406 & -131 & 212 & $-1,000$ & $-1,034$ \\
\hline Errors and Omissions and Other Capital & -856 & -416 & $-1,720$ & -944 & $-1,975$ \\
\hline Overall Balance & 1,338 & 314 & 3,122 & 4,637 & 15,422 \\
\hline Reserve items & $-1,338$ & -314 & $-3,122$ & -6146 & -15422 \\
\hline \multicolumn{6}{|l|}{ Memorandum items: } \\
\hline Official exchange rate, LD/US\$ (pa) & 0.61 & 1.27 & 1.28 & 1.30 & 1.31 \\
\hline Official exchange rate, LD/US\$ (eop) & 0.65 & 1.21 & 1.30 & 1.24 & 1.35 \\
\hline Gross official reserves (in billions of US\$) & 39.3 & 39.3 & 39.3 & 39.3 & 39.3 \\
\hline Gross official reserves, in months of next year's imports of GS & 17.6 & 19.4 & 21.9 & 23.3 & 30.2 \\
\hline Current account balance (in percent of GDP) & 13.3 & 3.3 & 21.5 & 24.3 & 41.6 \\
\hline Overall balance of payments (in percent of GDP) & 4.2 & 1.6 & 13.0 & 15.2 & 37.0 \\
\hline Nominal GDP (in billions of US\$) & 32.2 & 19.8 & 24.0 & 30.5 & 41.7 \\
\hline
\end{tabular}

Source: Central Bank of Libya; and staff estimates and projections.

$1 /$ Includes foreign partners' oil share.

2/ Includes partners' profit remittances from oil investment.

3/ For 2003 and 2004, includes payments for the Lockerbie Settlement of US $\$ 1,076$ million and US $\$ 1,080$ million, respectively. 
Table 30. Libya: Composition of External Trade , 2001-05

\begin{tabular}{lrrrrr}
\multicolumn{7}{c}{ (In percent of total) } & & & & \\
\hline & 2001 & 2002 & 2003 & 2004 & 2005 \\
\hline Imports 1/ & 100.0 & 100.0 & 100.0 & 100.0 & 100.0 \\
Food and live animals & 17.1 & 15.0 & 13.4 & 14.1 & 14.8 \\
Beverages and tobacco & 0.4 & 0.4 & 0.1 & 0.2 & 0.4 \\
Crude materials, inedible, except fuels & 2.2 & 2.1 & 1.4 & 1.4 & 1.8 \\
Mineral fuels, lubricants, and related materials & 0.1 & 1.3 & 0.7 & 0.7 & 0.3 \\
Animal and vegetable oils and fats & 1.9 & 0.9 & 3.1 & 1.9 & 1.4 \\
Chemicals & 6.5 & 8.5 & 5.3 & 4.0 & 5.9 \\
Materials & 15.9 & 19.8 & 20.9 & 19.9 & 20.7 \\
Machinery and transport equipment & 42.3 & 43.0 & 48.0 & 48.0 & 47.6 \\
Miscellaneous manufactures & 13.6 & 9.0 & 7.1 & 9.8 & 7.1 \\
Exports 2/ & 100.0 & 100.0 & 100.0 & 100.0 & 100.0 \\
Hydrocarbons & 96.1 & 98.1 & 96.6 & 95.7 & 97.3 \\
Other & 3.9 & 1.9 & 3.4 & 4.3 & 2.7 \\
\hline
\end{tabular}

Source: Census and Statistics Department; and staff estimates.

1/ Based on customs statistics.

2/ Based on balance of payments data. 
Table 31. Libya: Direction of Trade, 2001-05 1/

(In percent of total )

\begin{tabular}{|c|c|c|c|c|c|}
\hline & 2001 & 2002 & 2003 & 2004 & 2005 \\
\hline Imports & 100.0 & 100.0 & 100.0 & 100.0 & 100.0 \\
\hline Arab countries & 10.2 & 9.7 & 10.1 & 6.1 & 8.7 \\
\hline Asian countries & 14.5 & 18.5 & 13.8 & 22.9 & 21.7 \\
\hline Of which: Japan & 3.5 & 6.5 & 8.7 & 8.3 & 3.1 \\
\hline Other African countries & 0.9 & 0.4 & 0.4 & 0.2 & 0.3 \\
\hline European countries & 67.9 & 63.3 & 71.8 & 63.1 & 61.1 \\
\hline \multicolumn{6}{|l|}{ Of which: } \\
\hline France & 4.4 & 4.4 & 4.9 & 4.0 & 5.2 \\
\hline Germany & 13.1 & 11.2 & 8.9 & 12.0 & 9.3 \\
\hline Greece & 0.4 & 0.8 & 1.0 & 0.6 & 0.8 \\
\hline Italy & 24.3 & 24.4 & 18.4 & 18.3 & 11.9 \\
\hline Netherlands & 2.6 & 1.8 & 1.3 & 1.1 & 1.0 \\
\hline Spain & 1.8 & 1.9 & 1.4 & 1.5 & 1.0 \\
\hline Turkey & 1.3 & 1.5 & 2.1 & 1.8 & 2.0 \\
\hline United Kingdom & 5.7 & 7.0 & 5.4 & 4.1 & 3.4 \\
\hline Western Hemisphere countries & 5.8 & 7.2 & 3.5 & 7.5 & 7.8 \\
\hline \multicolumn{6}{|l|}{ Of which: } \\
\hline Canada & 0.6 & 1.1 & 1.1 & 1.8 & 0.8 \\
\hline United States & 2.8 & 2.9 & 1.9 & 2.5 & 3.0 \\
\hline Unallocated & 0.7 & 0.8 & 0.4 & 0.2 & 0.4 \\
\hline Exports & 100.0 & 100.0 & 100.0 & 100.0 & 100.0 \\
\hline Arab countries & 6.6 & 6.2 & 4.6 & 3.9 & 3.6 \\
\hline Asian countries & 2.8 & 3.3 & 4.9 & 4.1 & 15.1 \\
\hline Other African countries & 0.3 & 0.4 & 0.2 & 0.2 & 0.1 \\
\hline European countries & 90.0 & 89.9 & 89.9 & 90.5 & 76.8 \\
\hline \multicolumn{6}{|l|}{ Of which: } \\
\hline France & 3.6 & 3.7 & 2.6 & 2.1 & 5.6 \\
\hline Germany & 14.1 & 13.3 & 14.7 & 18.3 & 9.7 \\
\hline Greece & 2.3 & 2.4 & 2.2 & 2.1 & 3.2 \\
\hline Italy & 41.6 & 41.0 & 39.6 & 39.3 & 41.5 \\
\hline Netherlands & 1.2 & 1.1 & 0.9 & 0.7 & 2.0 \\
\hline Spain & 15.5 & 17.1 & 14.7 & 13.3 & 10.0 \\
\hline Turkey & 6.2 & 5.8 & 7.7 & 8.3 & 7.5 \\
\hline United Kingdom & 1.8 & 2.8 & 2.4 & 1.7 & 2.0 \\
\hline Western Hemisphere countries & 0.3 & 0.2 & 0.4 & 1.3 & 4.4 \\
\hline
\end{tabular}

Source: Census and Statistics Department.

1/ Based on customs statistics, which differ from balance of payments data in both timing and coverage. 
Table 32. Libya: Summary of the Tax System

Updated: November 2006

\begin{tabular}{|c|c|c|c|}
\hline \multicolumn{4}{|c|}{$\begin{array}{l}\text { 1. TAXES ON INCOME, PROFITS, AND CAPITAL GAINS } \\
\text { A. Individuals }\end{array}$} \\
\hline Tax & Nature of Tax & Exemptions and Deductions & Rates \\
\hline $\begin{array}{l}\text { 1. TAX ON } \\
\text { AGRICULTURAL } \\
\text { INCOME }\end{array}$ & $\begin{array}{l}\text { A tax is levied } \\
\text { on income from } \\
\text { the pure } \\
\text { agricultural } \\
\text { exploitation of } \\
\text { agricultural } \\
\text { land. }\end{array}$ & $\begin{array}{l}\text { Income generated from the first } 10 \\
\text { years from the Law's entry into force. } \\
\text { Personal allowances: } \\
\text { (a) single person LD } 1,200 \\
\text { (b) married person with no dependent } \\
\text { children LD } 1,800 \\
\text { (c) married person, widowed, or } \\
\text { divorced, with dependent children } \\
\text { LD } 2,400 \text {. } \\
\text { In addition: (a) life assurance } \\
\text { premiums on the life of the taxpayer, } \\
\text { his wife, or dependents up to a } \\
\text { maximum of LD } 600 \text { annually; (b) } \\
\text { premiums on general insurance up to } \\
\text { LD } 420 \text { annually. }\end{array}$ & A flat rate of 5 percent. \\
\hline $\begin{array}{l}\text { 2. TAX ON } \\
\text { INDUSTRIAL AND } \\
\text { COMMERCIAL } \\
\text { PROFITS }\end{array}$ & $\begin{array}{l}\text { Income } \\
\text { resulting from } \\
\text { any } \\
\text { commercial, } \\
\text { industrial, or } \\
\text { trade activities } \\
\text { are subject to } \\
\text { this tax. }\end{array}$ & $\begin{array}{l}\text { Personal allowances: } \\
\text { (a) single person LD } 1,200 \\
\text { (b) married person with no dependent } \\
\text { children LD } 1,800 \\
\text { (c) married person, widowed, or } \\
\text { divorced, with dependent children } \\
\text { LD } 2,400 \text {. } \\
\text { In addition: (a) life insurance } \\
\text { premiums on the life of the taxpayer, } \\
\text { his wife, or dependents up to a } \\
\text { maximum of LD } 600 \text { annually; (b) } \\
\text { premiums on general insurance up to } \\
\text { LD } 420 \text { annually. }\end{array}$ & 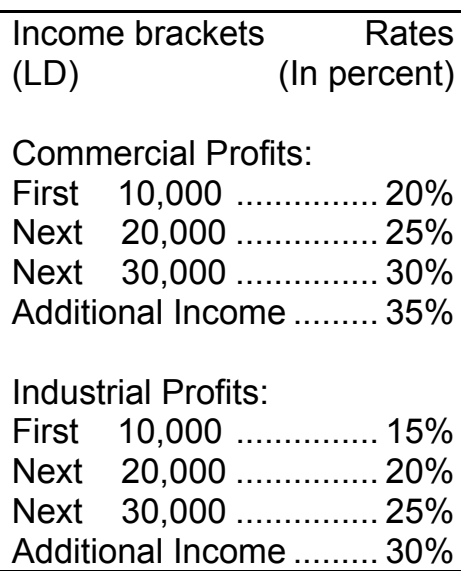 \\
\hline $\begin{array}{l}\text { 3. TAX ON } \\
\text { INCOME FROM } \\
\text { ABROAD }\end{array}$ & $\begin{array}{l}\text { Income from } \\
\text { abroad of } \\
\text { Libyan and } \\
\text { foreign } \\
\text { residents in the } \\
\text { country are } \\
\text { subject to this } \\
\text { tax. }\end{array}$ & $\begin{array}{l}\text { Salaries, wages, and similar income } \\
\text { received for work performed abroad } \\
\text { are exempt. }\end{array}$ & A flat rate of 20 percent. \\
\hline $\begin{array}{l}\text { 4. TAX ON } \\
\text { INTEREST } \\
\text { INCOME }\end{array}$ & $\begin{array}{l}\text { Interest } \\
\text { resulting from } \\
\text { deposits with } \\
\text { banks is } \\
\text { subject to this } \\
\text { tax. }\end{array}$ & Savings accounts are exempt. & A flat rate of 5 percent \\
\hline
\end{tabular}




\begin{tabular}{|c|c|c|c|}
\hline Tax & Nature of Tax & Exemptions and Deductions & Rates \\
\hline $\begin{array}{l}\text { 5. TAX ON } \\
\text { INCOME IN } \\
\text { ENTITIES } \\
\text { THAT APPLY } \\
\text { THE PHRASE } \\
\text { "PARTNERS, } \\
\text { NOT } \\
\text { EMPLOYEES" }\end{array}$ & $\begin{array}{l}\text { The income of } \\
\text { partners in all } \\
\text { production } \\
\text { entities that apply } \\
\text { the phrase } \\
\text { "partners, not } \\
\text { employees" is } \\
\text { subject to this } \\
\text { tax. }\end{array}$ & $\begin{array}{l}\text { Personal allowances: } \\
\text { (a) single person LD } 1,200 \\
\text { (b) married person with no dependent } \\
\text { children LD } 1,800 \\
\text { (c) married person, widowed, or } \\
\text { divorced, with dependent children } \\
\text { LD } 2,400 \text {. } \\
\text { In addition: (a) life assurance } \\
\text { premiums on the life of the taxpayer, } \\
\text { his wife, or dependents up to a } \\
\text { maximum of LD } 600 \text { annually; (b) } \\
\text { premiums on general insurance up to } \\
\text { LD } 420 \text { annually. } \\
\text { Others: a) depreciation on all assets } \\
\text { used to generate income; (b) bad } \\
\text { debt; (c) sums paid under the social } \\
\text { security system, or other approved } \\
\text { schemes not to exceed } 10 \text { percent of } \\
\text { the total collected; (d) taxes and fees } \\
\text { paid in connection with the activity } \\
\text { taxed; (e) contributions to government } \\
\text { recognized non profit charitable } \\
\text { entities, not in excess of } 2 \text { percent of } \\
\text { net income. }\end{array}$ & 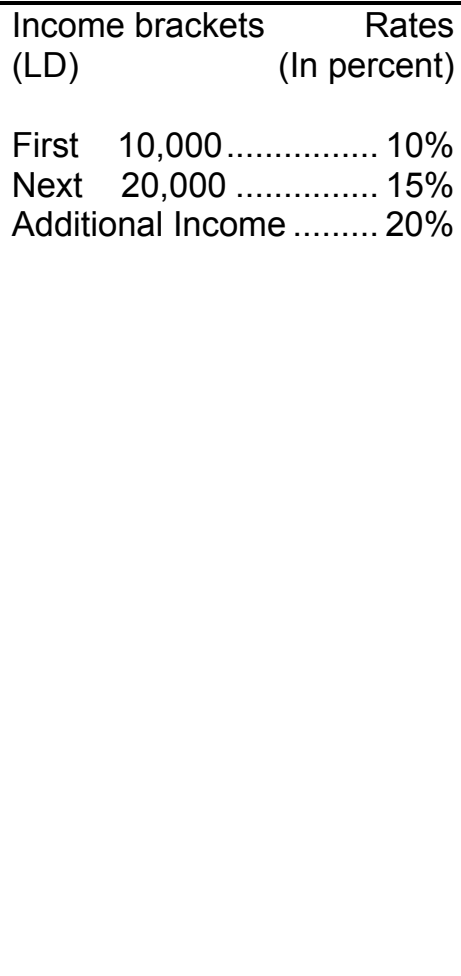 \\
\hline $\begin{array}{l}\text { 6. TAX ON } \\
\text { NONCOMMER } \\
\text { CIAL PROFITS }\end{array}$ & $\begin{array}{l}\text { This tax applies } \\
\text { to income from } \\
\text { the liberal } \\
\text { professions. }\end{array}$ & $\begin{array}{l}\text { Personal allowances: } \\
\text { (a) single person LD } 1,200 \\
\text { (b) married person with no dependent } \\
\text { children LD } 1,800 \\
\text { (c) married person, widowed, or } \\
\text { divorced, with dependent children } \\
\text { LD } 2,400 \text {. } \\
\text { In addition: (a) life assurance } \\
\text { premiums on the life of the taxpayer, } \\
\text { his wife, or dependents up to a } \\
\text { maximum of LD } 600 \text { annually; (b) } \\
\text { premiums on general insurance up to } \\
\text { LD } 420 \text { annually. } \\
\text { Others: a) depreciation on all assets } \\
\text { used to generate income; (b) bad } \\
\text { debt; (c) sums paid under the social } \\
\text { security system, or other approved } \\
\text { schemes not to exceed } 10 \text { percent of } \\
\text { the total collected; (d) taxes and fees } \\
\text { paid in connection with the activity } \\
\text { taxed; (e) contributions to government } \\
\text { recognized non profit charitable } \\
\text { entities, not in excess of } 2 \text { percent of } \\
\text { net income. }\end{array}$ & 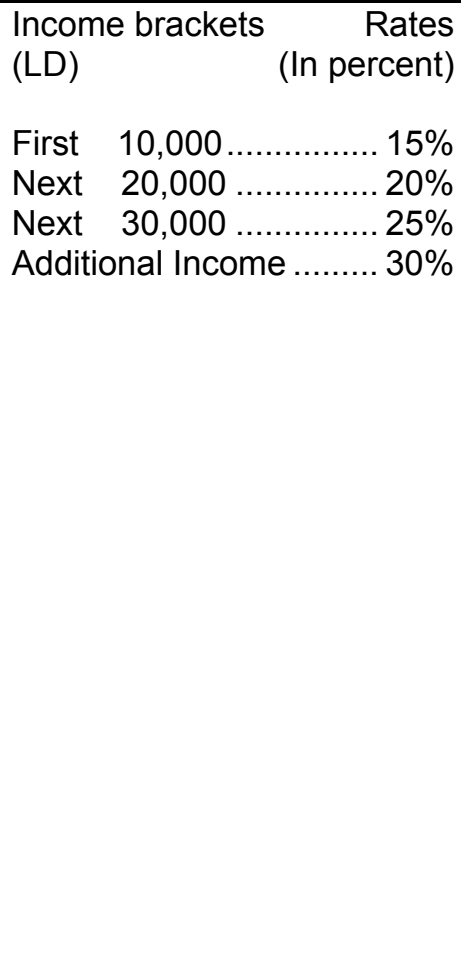 \\
\hline
\end{tabular}




\begin{tabular}{|c|c|c|c|}
\hline Tax & Nature of Tax & Exemptions and Deductions & Rates \\
\hline $\begin{array}{l}\text { 7. TAX ON } \\
\text { WAGES AND } \\
\text { SALARIES }\end{array}$ & $\begin{array}{l}\text { This tax applies } \\
\text { to income from } \\
\text { labor and any } \\
\text { income from any } \\
\text { service or } \\
\text { function, whether } \\
\text { of a permanent } \\
\text { or temporary } \\
\text { nature. } \\
\text { The tax is } \\
\text { deducted at } \\
\text { source by the } \\
\text { employer. }\end{array}$ & $\begin{array}{l}\text { Personal allowances: } \\
\text { (a) single person LD 1,200 } \\
\text { (b) married person with no dependent } \\
\text { children LD } 1,800 \\
\text { (c) married person, widowed, or } \\
\text { divorced, with dependent children } \\
\text { LD } 2,400 \text {. } \\
\text { In addition: (a) contributions to social } \\
\text { security, or other approved schemes; } \\
\text { (b) amounts received as } \\
\text { reimbursement for expenses incurred } \\
\text { in performing duties; (c) deductions or } \\
\text { fines; (d) monetary allowances for } \\
\text { leave accumulated at end of service. } \\
\text { Others: a) depreciation on all assets } \\
\text { used to generate income; (b) bad } \\
\text { debt; (c) sums paid under the social } \\
\text { security system, or other approved } \\
\text { schemes not to exceed } 10 \text { percent of } \\
\text { the total collected; (d) taxes and fees } \\
\text { paid in connection with the activity } \\
\text { taxed; (e) contributions to government } \\
\text { recognized non profit charitable } \\
\text { entities, not in excess of } 2 \text { percent of } \\
\text { net income. }\end{array}$ & $\begin{array}{lr}\begin{array}{l}\text { Income brackets } \\
\text { (LD) }\end{array} & \begin{array}{r}\text { Rates } \\
\text { (In percent) }\end{array} \\
& \\
\text { First } & 4,800 \ldots \ldots \ldots . . .8 \% \\
\text { Next } & 4,800 \ldots \ldots . .10 \% \\
\text { Additional Income } \ldots \ldots \ldots . . .15 \%\end{array}$ \\
\hline 8. JIHAD TAX & $\begin{array}{l}\text { An additional tax } \\
\text { levied on all } \\
\text { taxable income. }\end{array}$ & None. & 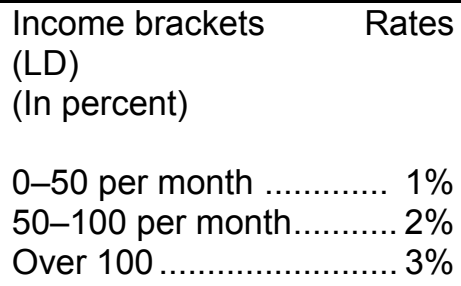 \\
\hline \multicolumn{4}{|c|}{ B. CORPORATION } \\
\hline $\begin{array}{l}\text { 1. TAX ON } \\
\text { COMPANIES }\end{array}$ & $\begin{array}{l}\text { Companies and } \\
\text { branches of } \\
\text { foreign } \\
\text { companies in } \\
\text { Libya are subject } \\
\text { to this tax. } \\
\text { Petroleum } \\
\text { companies are } \\
\text { subject to special } \\
\text { provisions } \\
\text { concerning } \\
\text { royalties, income } \\
\text { tax, and } \\
\text { production } \\
\text { sharing. }\end{array}$ & $\begin{array}{l}\text { Establishment expenses as defined } \\
\text { by the Law. }\end{array}$ & 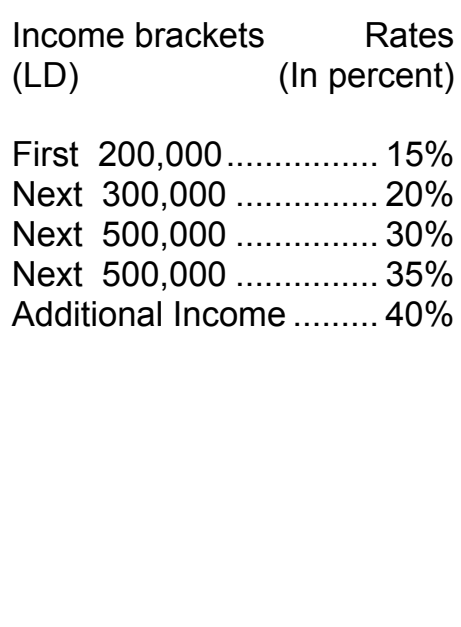 \\
\hline
\end{tabular}




\begin{tabular}{|c|c|c|c|}
\hline \multicolumn{4}{|c|}{ 2. TAXES ON GOODS AND SERVICES } \\
\hline Tax & Nature of Tax & Exemptions and Deductions & Rates \\
\hline $\begin{array}{l}\text { 1. GENERAL } \\
\text { SALES TAX }\end{array}$ & Does not exist. & None. & None. \\
\hline 2. EXCISES & $\begin{array}{l}\text { The excise taxes } \\
\text { apply to a } \\
\text { number of } \\
\text { commodities } \\
\text { whether they are } \\
\text { domestically- } \\
\text { produced or } \\
\text { imported. } \\
\text { Taxes on } \\
\text { petroleum } \\
\text { products are } \\
\text { specific and are } \\
\text { the same on both } \\
\text { imports and } \\
\text { domestic } \\
\text { production. } \\
\text { Taxes on all } \\
\text { other } \\
\text { commodities are } \\
\text { ad valorem and } \\
\text { are lower on } \\
\text { domestic } \\
\text { production as a } \\
\text { protective } \\
\text { measure. }\end{array}$ & None. & $\begin{array}{l}\text { For petroleum products, the } \\
\text { taxes are in the range of } 7- \\
32 \text { dirhams per liter. } \\
\text { For other commodities, the } \\
\text { rates are in the range of } 2- \\
5 \text { percent on domestic } \\
\text { production (Production Tax) } \\
\text { and } 15-25 \text { percent on } \\
\text { imports (Consumption Tax). }\end{array}$ \\
\hline \multicolumn{4}{|c|}{ 3. TAXES ON INTERNATIONAL TRADE } \\
\hline $\begin{array}{l}\text { 1. IMPORT } \\
\text { DUTIES }\end{array}$ & $\begin{array}{l}\text { Duties are levied } \\
\text { on imports } \\
\text { classified } \\
\text { according to } \\
\text { Brussels } \\
\text { classification. }\end{array}$ & None. & $\begin{array}{l}\text { There are two import duty } \\
\text { rates: } 10 \text { percent for tobacco } \\
\text { products and zero for all } \\
\text { other goods. }\end{array}$ \\
\hline $\begin{array}{l}\text { 2. EXPORT } \\
\text { TAXES }\end{array}$ & $\begin{array}{l}\text { Duties are levied } \\
\text { on a small } \\
\text { number of } \\
\text { agricultural } \\
\text { products, textiles, } \\
\text { precious metals, } \\
\text { and medicines. }\end{array}$ & $\begin{array}{l}\text { Exports of public corporations are } \\
\text { exempt. }\end{array}$ & $\begin{array}{l}\text { Export taxes are specific for } \\
\text { agricultural products and } \\
\text { range from LD } 100 \text { to } L D \\
1,500 \text { per kilo. Exports of } \\
\text { manufactured products are } \\
\text { subject to a } 50 \text { percent tax. }\end{array}$ \\
\hline \multicolumn{4}{|c|}{ 4. SOCIAL SECURITY } \\
\hline $\begin{array}{l}\text { 1. SOCIAL } \\
\text { SECURITY }\end{array}$ & $\begin{array}{l}\text { Applies to wages } \\
\text { and salaries. }\end{array}$ & None. & $\begin{array}{l}\text { Employees contribute } \\
3.75 \text { percent and employers } \\
11.25 \text { percent. }\end{array}$ \\
\hline
\end{tabular}




\begin{tabular}{|c|c|c|c|}
\hline \multicolumn{4}{|c|}{ 5. Other TAXES } \\
\hline Tax & Nature of Tax & Exemptions and Deductions & Rates \\
\hline $\begin{array}{l}\text { 1. STAMP } \\
\text { DUTIES }\end{array}$ & $\begin{array}{l}\text { The duty is levied } \\
\text { on a range of } \\
\text { instruments and } \\
\text { transactions } \\
\text { including bills of } \\
\text { exchange, } \\
\text { leases, contracts, } \\
\text { mortgages, and } \\
\text { licenses. }\end{array}$ & $\begin{array}{l}\text { Documents and actions drawn up or } \\
\text { concluded by public agencies. } \\
\text { Documents and actions drawn up or } \\
\text { concluded by government-recognized } \\
\text { organizations for professional, social, } \\
\text { cultural, charitable, or athletic } \\
\text { activities; or to serve the public } \\
\text { interest. } \\
\text { Documents and actions drawn up or } \\
\text { concluded by foreign diplomatic or } \\
\text { consular entities, subject to } \\
\text { reciprocity. } \\
\text { Documents and actions drawn up or } \\
\text { concluded by certain international } \\
\text { organizations. } \\
\text { Documents related to Pilgrimage to } \\
\text { Mecca. } \\
\text { Documents related to studies in } \\
\text { various educational institutions. } \\
\text { Documents or actions drawn up or } \\
\text { concluded by persons drawing basic } \\
\text { pensions or wages. } \\
\text { Documents submitted by persons } \\
\text { seeking employment. } \\
\text { Other exemptions specific to each } \\
\text { stamp duty also apply. }\end{array}$ & $\begin{array}{l}\text { The duty is specific to each } \\
\text { action as stipulated by its } \\
\text { Law. }\end{array}$ \\
\hline $\begin{array}{l}\text { 2. THE } \\
\text { GREAT MAN- } \\
\text { MADE RIVER }\end{array}$ & $\begin{array}{l}\text { This is an extra- } \\
\text { budgetary tax } \\
\text { levied on sales of } \\
\text { gasoline, diesel, } \\
\text { cigarettes, and } \\
\text { airline tickets. }\end{array}$ & None. & $\begin{array}{l}\text { Item } \\
\text { Gates } \\
\text { Gasoline (per liter).LD } 0.020 \\
\text { Diesel (per liter) .....LD } 0.005 \\
\text { Cigarettes (per cigarette).LD } \\
0.010 \\
\text { Airline tickets................ } 10 \%\end{array}$ \\
\hline
\end{tabular}

Sources: Various published sources. 